\title{
Development of a new quantitative structure-activity relationship model for predicting Ames mutagenicity of food flavor chemicals using StarDrop ${ }^{\mathrm{TM}}$ auto- Modeller ${ }^{\mathrm{TM}}$
}

Toshio Kasamatsu', Airi Kitazawa', Sumie Tajima², Masahiro Kaneko², Kei-ichi Sugiyama', Masami Yamada1,3, Manabu Yasui ${ }^{1}$, Kenichi Masumura', Katsuyoshi Horibata ${ }^{1}$ and Masamitsu Honma ${ }^{1,4^{*}}$ (i)

\begin{abstract}
Background: Food flavors are relatively low molecular weight chemicals with unique odor-related functional groups that may also be associated with mutagenicity. These chemicals are often difficult to test for mutagenicity by the Ames test because of their low production and peculiar odor. Therefore, application of the quantitative structure-activity relationship (QSAR) approach is being considered. We used the StarDrop ${ }^{\text {TM }}$ Auto-Modeller ${ }^{\text {TM }}$ to develop a new QSAR model.

Results: In the first step, we developed a new robust Ames database of 406 food flavor chemicals consisting of existing Ames flavor chemical data and newly acquired Ames test data. Ames results for some existing flavor chemicals have been revised by expert reviews. We also collected 428 Ames test datasets for industrial chemicals from other databases that are structurally similar to flavor chemicals. A total of 834 chemicals' Ames test datasets were used to develop the new QSAR models. We repeated the development and verification of prototypes by selecting appropriate modeling methods and descriptors and developed a local QSAR model. A new QSAR model "StarDrop NIHS 834_67" showed excellent performance (sensitivity: 79.5\%, specificity: 96.4\%, accuracy: 94.6\%) for predicting Ames mutagenicity of 406 food flavors and was better than other commercial QSAR tools.
\end{abstract}

Conclusions: A local QSAR model, StarDrop NIHS 834_67, was customized to predict the Ames mutagenicity of food flavor chemicals and other low molecular weight chemicals. The model can be used to assess the mutagenicity of food flavors without actual testing.

Keywords: Quantitative structure-activity relationship (QSAR), Food flavors, Mutagenicity Ames test, StarDrop ${ }^{\mathrm{TM}}$ auto-Modeller ${ }^{\mathrm{TM}}$, Machine learning

\footnotetext{
* Correspondence: honma@nihs.go.jp

'Division of Genetics and Mutagenesis, National Institute of Health Sciences, Kawasaki city, Kanagawa, Japan

${ }^{4}$ Division of General Affairs, National Institute of Health Sciences, Kawasaki

City, Kanagawa, Japan

Full list of author information is available at the end of the article
}

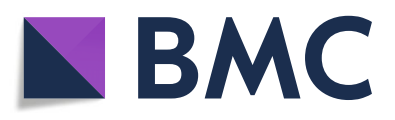

(- The Author(s). 2021 Open Access This article is licensed under a Creative Commons Attribution 4.0 International License, which permits use, sharing, adaptation, distribution and reproduction in any medium or format, as long as you give appropriate credit to the original author(s) and the source, provide a link to the Creative Commons licence, and indicate if changes were made. The images or other third party material in this article are included in the article's Creative Commons licence, unless indicated otherwise in a credit line to the material. If material is not included in the article's Creative Commons licence and your intended use is not permitted by statutory regulation or exceeds the permitted use, you will need to obtain permission directly from the copyright holder. To view a copy of this licence, visit http://creativecommons.org/licenses/by/4.0/. The Creative Commons Public Domain Dedication waiver (http://creativecommons.org/publicdomain/zero/1.0/) applies to the data made available in this article, unless otherwise stated in a credit line to the data. 


\section{Introduction}

Food flavor chemicals are used and/or present in foods at very low level. Human exposure to these flavor chemicals through foods is too low to raise concerns about general toxicity. Regarding mutagenicity, however, there are health concerns even with trace amounts because there is no threshold for mutagenicity, and even very low levels of exposure of mutagenic chemicals do not result in zero carcinogenic risk [1]. Therefore, the presence or absence of mutagenicity is an important point for risk assessment of flavor chemicals.

The bacterial reverse mutation test (Ames test) is an important mutagenicity test, but it requires approximately $2 \mathrm{~g}$ of sample for a dose-finding study and main study [2]. On the other hand, the amount of flavor produced industrially is extremely small, which often means that testing is impossible. Additionally, the peculiar odor of some flavors sometimes makes it difficult to perform the test in the laboratory. Recently, quantitative structure-activity relationship (QSAR) approaches instead of the Ames test have been frequently used for assessing the mutagenicity of chemicals [3]. Ono et al. assessed the viability of QSAR tools by using three QSAR tools to calculate the Ames mutagenicity of 367 flavor chemicals (for which Ames test results were available) [4]. Consequently, the highest sensitivity (the ability of a QSAR tool to detect Ames positives chemicals correctly) was $38.9 \%$ with the single tool and $47.2 \%$ even with the combination of three tools, which indicated that application of QSAR tools to assess the Ames mutagenicity of flavor chemicals was still premature. Therefore, it is necessary to improve or develop QSAR tools for predicting Ames mutagenicity of flavor chemicals.

Flavor chemicals are relatively low molecular weight chemical substances mainly composed of carbon, hydrogen, oxygen, nitrogen, and sulfur that often have specific functional groups. In Japan, most food flavors are classified into 18 types according to their chemical structure [5]. Therefore, with a focus on their characteristic chemical space, we thought that there was potential to increase the predictive performance by developing a local QSAR model customized for flavor chemicals. In recent years, computational software has been provided to assist with development of QSAR models by machine learning. We have tried to develop a QSAR model specialized for flavor chemicals using StarDrop ${ }^{\text {ma }}$ software, which has a module (Auto-Modeller ${ }^{\mathrm{mw}}$ ) that can generate predictive models automatically.

Before developing the QSAR model, we developed a new robust Ames database of 406 food flavor chemicals that is based on Ono's database [4]. We re-evaluated ambiguous data judged as "equivocal" in Ono's database via literature review and incorporated Ames test data of flavor chemicals from other publicly available databases.
In parallel, we performed the Ames test with key flavor chemicals of which Ames data is unknown and incorporated their results into the new database. This benchmark food flavor chemical database is useful for development of QSAR models and evaluation of QSAR model performance.

\section{Materials \& methods}

Ames test database of food flavor chemicals

We utilized the Ames test database of food flavor chemicals reported by Ono et al. [4], but because the database includes 14 "equivocal" judgments (Table 1), we reevaluated by reviewing the reference literature and reclassified them as positive, negative, or inconclusive. Ames test data of the "inconclusive" chemicals were excluded from the database. If there were any other flavor chemicals from publicly available Ames test database (Hansen database [6]), they were also added.

\section{Ames test}

Ames tests were performed for 45 flavor chemicals. The purities and suppliers of the test chemicals are shown in Table 2. The Ames tests were conducted by contract research organizations following Good Laboratory Practice compliance according to the Industrial Safety and Health Act test guideline with preincubation method [7]. The test guideline requires five strains (Salmonella thyphimurium TA100, TA98, TA1535, TA1537, and Escherichia coli WP2 uvrA) under both the presence and absence of metabolic activation (rat S9 mix prepared from phenobarbital and 5,6-benzoflavone-induced rat liver), which is similar to the Organization of Economic Co-operation and Development guideline TG471 [8]. The positive criterion is when the number of revertant colonies increased more than twice as much as the control in at least one Ames test strain in the presence or absence of S9 mix. Dose dependency and reproducibility were also considered in the final judgment. The relative activity value (RAV), which is defined as the number of induced revertant colonies per $\mathrm{mg}$, was calculated for the positive result.

\section{Commercial QSAR tools}

DEREK Nexus ${ }^{\text {TM }}$ is a knowledge-based commercial software developed by Lhasa Limited, UK $[9,10]$. The software includes knowledge rules created by considering insights related to structural alert, chemical compound examples, and metabolic activations and mechanisms. We used DEREK Nexus ${ }^{\text {Tx }}$ version 6.1.0 in this study. DEREK Nexus ${ }^{\mathrm{Tw}}$ ranks the possibility of mutagenicity (certain, probable, plausible, equivocal, doubted, improbable, impossible, open, contradicted, nothing to report) by applying a "reasoning rule." When it is "certain," 
Table 1 Re-evaluation of Ames test data, which were categorized as "equivocal" by Ono et al. [4]

\begin{tabular}{|c|c|c|c|c|c|c|}
\hline No. & $\begin{array}{l}\text { JECFA } \\
\text { No. }\end{array}$ & Chemical Name & CAS No. & $\begin{array}{l}\text { Judgement after } \\
\text { review }\end{array}$ & $\begin{array}{l}\text { Key } \\
\text { reference* }\end{array}$ & Comments \\
\hline 1 & 252 & isobutanal & $78-84-2$ & Negative & [13] & $\begin{array}{l}\text { The study condition did not } \\
\text { meet current standard. Other } \\
\text { available data indicative of negative. }\end{array}$ \\
\hline 2 & 690 & phenol & $108-95-2$ & Negative & [14] & $\begin{array}{l}\text { Only one positive report of which } \\
\text { response was weak. Other } \\
\text { available data indicative of negative. }\end{array}$ \\
\hline 3 & 738 & furfuryl alcohol & $98-00-0$ & Negative & [15] & $\begin{array}{l}\text { Only one report was positive among } \\
6 \text { reports reviewed in the key reference. } \\
\text { Although no detail was available, } \\
\text { the study conditon is unlikely } \\
\text { meet current standard. }\end{array}$ \\
\hline 4 & 744 & furfural & $98-01-1$ & Negative & [15] & $\begin{array}{l}\text { Among } 14 \text { reports reviewed in } \\
\text { the key reference, } 4 \text { reports } \\
\text { indicative of positive were questionable. } \\
\text { Other } 10 \text { reports were negative. }\end{array}$ \\
\hline 5 & 836 & 2-hydroxy-1,2-diphenylethanone & 119-53-9 & Inconclusive & [16] & $\begin{array}{l}\text { Weak positive. Other available data } \\
\text { are a mixture of positives/negatives. } \\
\text { No conclusion drawn. }\end{array}$ \\
\hline 6 & 1168 & 3-propylidenephthalide & $\begin{array}{l}17,369- \\
59-4\end{array}$ & Inconclusive & [17] & $\begin{array}{l}\text { One positive report reviewed in } \\
\text { the key reference raised a question } \\
\text { about purity. Other available data } \\
\text { were also unclear. }\end{array}$ \\
\hline 7 & 1172 & 6-methylcoumarin & $92-48-8$ & Negative & [18] & $\begin{array}{l}\text { Ambiguous response. Other } \\
\text { available data indicative of negative. }\end{array}$ \\
\hline 8 & 1342 & delta-3-carene & $\begin{array}{l}13,466- \\
78-9\end{array}$ & Inconclusive & [19] & $\begin{array}{l}\text { Positve though not meeting current } \\
\text { standard. Recent other data } \\
\text { (Saverni, 2012) indicative of negative. } \\
\text { No conclusion drawn. }\end{array}$ \\
\hline 9 & 1450 & 4-hydroxy-5-methyl-3(2H)-furanone & $\begin{array}{l}19,322- \\
27-1\end{array}$ & Positive & [20] & $\begin{array}{l}\text { Confirmed positive response. } \\
\text { No other data negate the } \\
\text { conclusion was available. }\end{array}$ \\
\hline 10 & 1481 & ethyl maltol & $4940-11-8$ & Inconclusive & [21] & $\begin{array}{l}\text { Two conflicting reports reviewed } \\
\text { in the key reference. } \\
\text { No conclusion drawn. }\end{array}$ \\
\hline 11 & 1560 & allyl isothiocyanate & $57-06-7$ & Positive & [22] & $\begin{array}{l}\text { Weak positive. Other available } \\
\text { data are a mixture of } \\
\text { positives/negatives. } \\
\text { "Isothiocyanate" structure } \\
\text { adopted as "positve alert" } \\
\text { in representative QSAR tools. }\end{array}$ \\
\hline 12 & 1561 & butyl isothiocyanate & $592-82-5$ & Positive & [23] & $\begin{array}{l}\text { Confirmed positive response. } \\
\text { No other data negate } \\
\text { the conclusion was available. }\end{array}$ \\
\hline 13 & 1563 & phenethyl isothiocyanate & $\begin{array}{l}2257-09- \\
2\end{array}$ & Positive & [22] & $\begin{array}{l}\text { Weak positive. Other available } \\
\text { data also indicate positive. }\end{array}$ \\
\hline 14 & 1776 & $\begin{array}{l}\text { ethyl 2-[(5-methyl-2-propan-2-yl } \\
\text { cyclohexanecarbonyl)amino]acetate }\end{array}$ & $\begin{array}{l}68,489- \\
14-5\end{array}$ & Negative & [15] & $\begin{array}{l}\text { Since the study report indicative } \\
\text { of weak positive reviewed in } \\
\text { the key reference was unpublished, } \\
\text { no reliability confirmed. Recent } \\
\text { GLP data submitted to MHLW under } \\
\text { ANEI-HOU was negative (undisclosed). }\end{array}$ \\
\hline
\end{tabular}

* Reference that was considered as a basis to draw a conclusion of "equivocal".

"probable," "plausible," or "equivocal," the query chemical is predicted to be positive in the Ames test.

CASE Ultra is a QSAR-based toxicity prediction software developed by MultiCASE Inc. (USA). CASE Ultra uses a statistical method to automatically extract alerts based on training data by using machine learning technology $[11,12]$. The structural characteristics of the alert surroundings are called the "modulator," and these are also learned automatically from the training data. In this algorithm, to construct a QSAR model with continuous toxicity endpoints, various physical chemistry parameters and descriptors are used. We used CASE Ultra version 1.8.0.2 with the GT1_BMUT module in this study. The prediction result of each module is ranked as "known positive," "positive," "negative," "known negative, " "inconclusive," or "out of domain." A query chemical ranked "known positive," "positive" or "inconclusive" is predicted to be positive in the Ames test. 


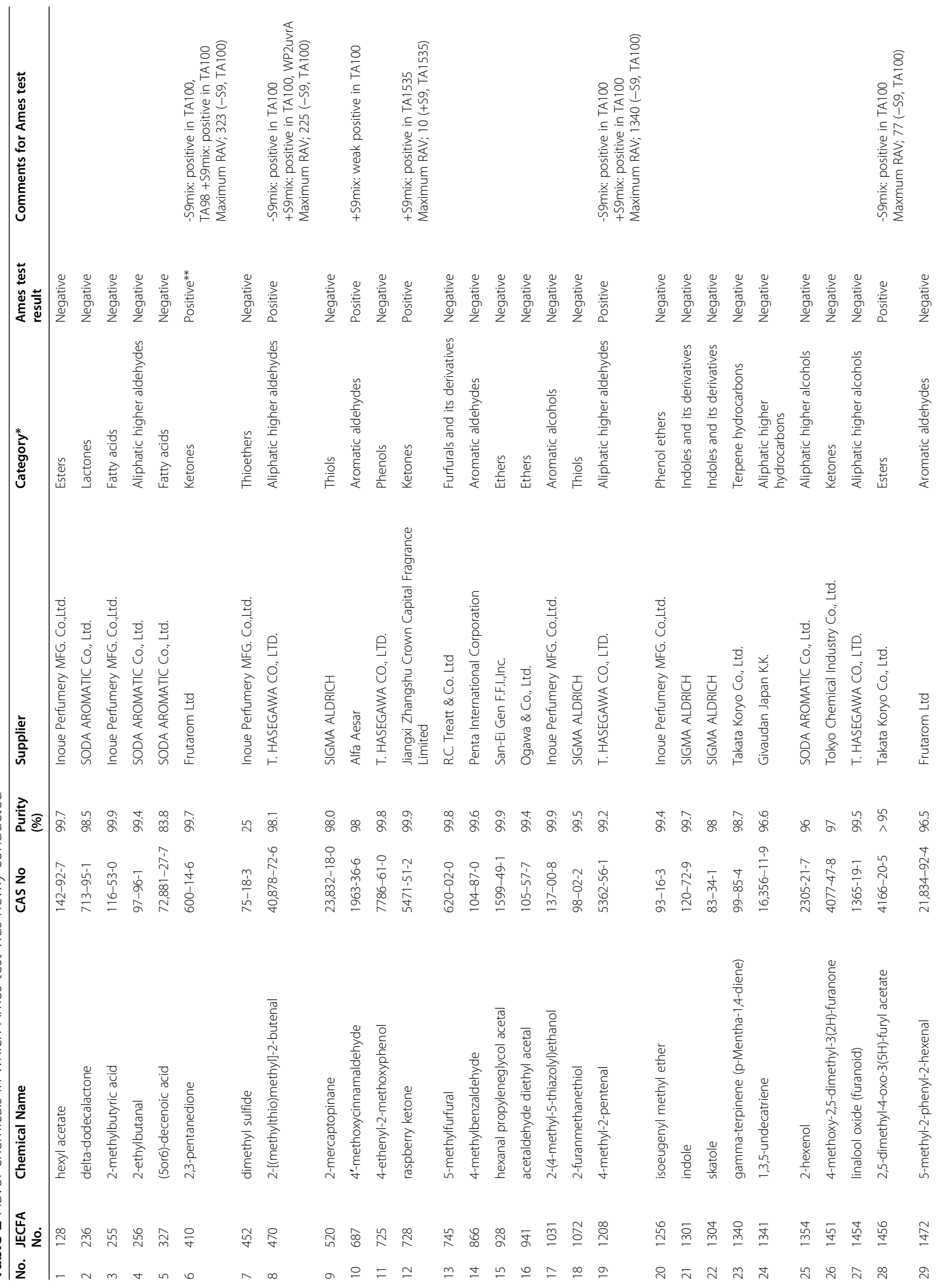




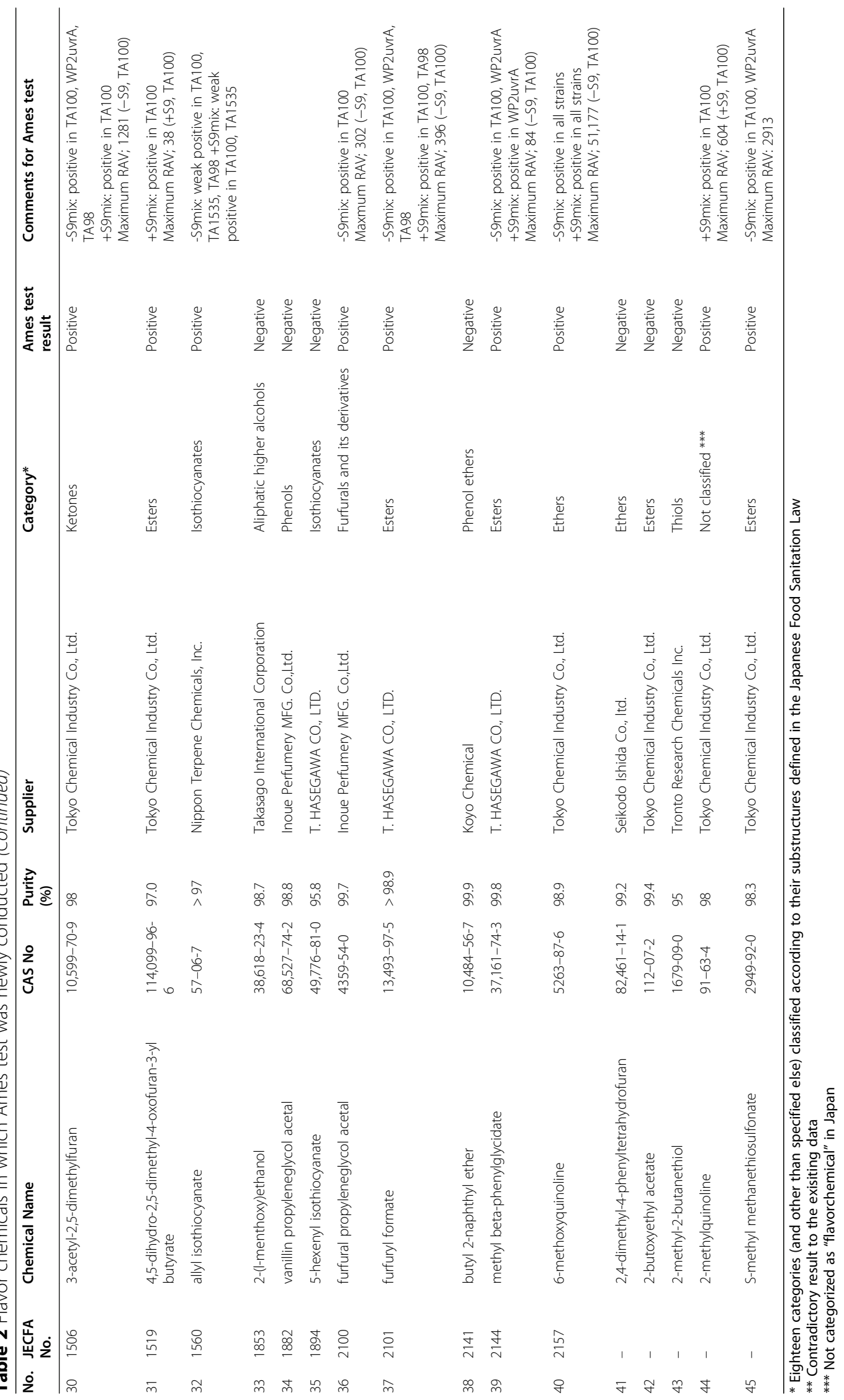


Table $32 \times 2$ contingency matrix for Ames mutagenicity classification

\begin{tabular}{llll}
\hline & \multicolumn{3}{c}{ QSAR prediction } \\
\hline Ames test result & & positive & negative \\
& positive & true positive (TP) & false negative (FN) \\
& negative & false positve (FP) & true negative (TN) \\
\hline
\end{tabular}

\section{Software for developing a new QSAR model}

StarDrop $^{\text {TM }}$ developed by Optibrium Ltd. (UK) is an integrated software for drug discovery that includes the statistics-based QSAR model generation tool, AutoModeller $^{\mathrm{Tm}}$. Using multiple modeling techniques and a suite of built-in descriptors, Auto-Modeller ${ }^{\text {Ts }}$ automatically generates tailored predictive models based on the study dataset for the domain that needs to be predicted.

\section{Analysis of QSAR tool performance}

Because the Ames test results are binary, positive, or negative, their predictive power can be objectively quantified and assessed from their coincidence from the QSAR calculation results. The $2 \times 2$ prediction matrix comprising true positive (TP), false positive (FP), false negative (FN), and true negative (TN) is given in Table 3. Sensitivity (ability to detect positive substances) is calculated as TP / $(\mathrm{TP}+\mathrm{FN})$, specificity (ability to detect negative substances) is calculated as $\mathrm{TN} /(\mathrm{TN}+\mathrm{FP})$, and accuracy (prediction rate of positive and negative) is calculated as $(\mathrm{TP}+\mathrm{TN}) /(\mathrm{TP}+\mathrm{TN}+\mathrm{FP}+\mathrm{FN})$. Applicability is provided by $(\mathrm{TP}+\mathrm{TN}+\mathrm{FP}+\mathrm{FN}) /$ total number.

\section{Results}

Development of a new Ames test database of food flavor chemicals

We developed a new Ames test database consisting of 406 food flavor chemicals (Table 4). The data source is described as follows.

Ono et al. reported an Ames test database consisting of 367 food flavor chemicals (positive: 24, equivocal: 12, negative: 331) [4]. However, it actually contained 369 chemicals (positive: 24, equivocal: 14, negative: 331). Table 1 shows the 14 equivocal chemicals. We reviewed key references that led to "equivocal" and re-evaluated to determine if there was evidence of positivity or negativity in view of current testing criteria. Our final judgment and the supporting reasons are described in Table 1 [13-23]. If there was insufficient evidence or no detailed information available for the judgment, we concluded that they were "inconclusive." Among 14 equivocal flavoring chemicals, four were positive, six were negative, and four were inconclusive. In total, 365 flavor chemicals (positive: 28, negative: 337), excluding four inconclusive chemicals, were added to the new database.

Two flavor chemicals, quinoline (91-22-5) and 4methylquinoline (491-35-0) have been added to the new database. Their Ames test data were found in the Hansen data set [6].

We newly performed Ames tests for 45 flavor chemicals. The information of tested samples and the Ames test results are shown in Table 2. Ten of the 45 Ames test results were previously reported [24]. The raw Ames test data are available in the Additional files. Among 45 flavor chemicals, 15 were positive and 30 were negative. Six chemicals, indole (120-72-9), 5-methylfurfural (620-02-0), 2,3-pentanedione (600-14-6), allyl isothiocyanate (57-06-7), skatole (83-34-1), and gammaterpinene (p-Mentha-1,4-diene) (99-85-4), are also present in Ono's database. In Ono's database [4], 2,3pentanedione was judged as negative, but it clearly increased the mutant frequency in TA100 in the absence of S9 mix (Additional file (6)). The results of these Ames tests are reflected in the new database. Finally, 39 new food flavor chemicals were added to the database.

\section{Development of a new QSAR model for predicting Ames mutagenicity}

We developed a new QSAR model for predicting Ames mutagenicity by using StarDrop ${ }^{\mathrm{TM}}$ Auto-Modeller ${ }^{\mathrm{TM}}$. To develop the QSAR model, the available Ames test study dataset is essential. We used 406 datasets of flavor chemicals in the new Ames test database to develop the model. To further increase the size of the dataset (especially positive data), we added Ames test data of chemicals structurally similar to flavor chemicals. We previously developed a large Ames test database consisting of $>12,000$ industrial chemicals [25]. We selected 428 chemicals (positive: 255; negative: 173) from the database that have molecular weights $<500$ and possess a characteristic substructure of flavor chemicals defined in the Food Sanitation Law in Japan [5]. The Ames test data of 834 chemicals (positive: 299, negative: 535 ) were integrated as the study dataset for the development of the QSAR model.

Prototypes of predictive models were built by using an automatic process. The study dataset was divided into training (70\%) and validation (30\%) data by using the cluster method, which uses an unsupervised nonhierarchical clustering algorithm developed by Butina [26]. Auto-Modeller ${ }^{\mathrm{rm}}$ has three modeling methods (Gaussian process, random forest, and decision tree) for the category model. In a pretest, the random forest model gave the best performance for our target. The descriptors were automatically generated, including whole molecule descriptors (e.g., molecular weight, logP, and polar surface area) and 2D structural descriptors from 
Table 4406 food flavor chemicals assessed by Ames test and QSARs Table $4 \mathbf{4 0 6}$ food flavor chemicals assessed by Ames test and QSARs

\begin{tabular}{|c|c|c|c|c|c|c|c|c|}
\hline No. & $\begin{array}{l}\text { JECFA } \\
\text { No. }\end{array}$ & Chemical name & CAS\# & Ames Result & $\begin{array}{c}\text { StarDrop } \\
\text { NIHS 834_67 }\end{array}$ & $\begin{array}{l}\text { Derek Nexus } \\
\quad 6.1 .0\end{array}$ & $\begin{array}{l}\text { CASE Ultra 1.8.0.2 } \\
\text { GT1_BMUT }\end{array}$ & Note* \\
\hline 1 & 217 & trans-anethole & $4180-23-8$ & Positive & Positive & INACTIVE & Known Negative & \\
\hline 2 & 408 & diacetyl & 431-03-8 & Positive & Positive & PLAUSIBLE & Known Negative & \\
\hline 3 & 410 & 2,3-pentadione & $600-14-6$ & Positive & Positive & PLAUSIBLE & Known Negative & $\begin{array}{l}\text { Ames test was newly conducted (Table 2). } \\
\text { Negative in Ono's data was revised. }\end{array}$ \\
\hline 4 & 429 & menthone & $89-80-5$ & Positive & Negative & INACTIVE & Known Negative & \\
\hline 5 & 470 & 2-[(methylthio)methyl]-2-butenal & $40878-72-6$ & Positive & Positive & PLAUSIBLE & Positive & Ames test was newly conducted (Table 2). \\
\hline 6 & 507 & methylsulfinylmethane & $67-68-5$ & Positive & Positive & INACTIVE & Known Positive & \\
\hline 7 & 656 & trans-cinnamaldehyde & $104-55-2$ & Positive & Negative & PLAUSIBLE & Known Positive & \\
\hline 8 & 687 & 4'-methoxycinnamaldehyde & $1963-36-6$ & Positive & Positive & PLAUSIBLE & Positive & Ames test was newly conducted (Table 2). \\
\hline 9 & 712 & resorcinol & $108-46-3$ & Positive & Positive & INACTIVE & Known Negative & \\
\hline 10 & 728 & raspberry ketone & 5471-51-2 & Positive & Negative & INACTIVE & Negative & Ames test was newly conducted (Table 2). \\
\hline 11 & 735 & 2-phenylphenol & $90-43-7$ & Positive & Positive & INACTIVE & Known Positive & \\
\hline 12 & 739 & furfuryl acetate & $623-17-6$ & Positive & Positive & PROBABLE & Known Positive & \\
\hline 13 & 767 & 2,6-dimethylpyrazine & $108-50-9$ & Positive & Negative & INACTIVE & Known Positive & \\
\hline 14 & 820 & 4-phenyl-3-buten-2-one & $122-57-6$ & Positive & Negative & INACTIVE & Known Positive & \\
\hline 15 & 937 & pyruvaldehyde & $78-98-8$ & Positive & Positive & PLAUSIBLE & Known Positive & \\
\hline 16 & 1032 & thiazole & $288-47-1$ & Positive & Positive & INACTIVE & Known Positive & \\
\hline 17 & 1147 & 1-penten-3-one & $1629-58-9$ & Positive & Positive & PROBABLE & Known Positive & \\
\hline 18 & 1175 & trans, trans-2,4-hexadienal & $142-83-6$ & Positive & Positive & PLAUSIBLE & Known Positive & \\
\hline 19 & 1208 & 4-methyl-2-pentenal & 5362-56-1 & Positive & Negative & PLAUSIBLE & Positive & Ames test was newly conducted (Table 2). \\
\hline 20 & 1302 & 6-methylquinoline & $91-62-3$ & Positive & Positive & PLAUSIBLE & Known Positive & \\
\hline 21 & 1307 & methyl 2-pyrrolyl ketone & $1072-83-9$ & Positive & Positive & INACTIVE & Known Positive & \\
\hline 22 & 1346 & cadinene (mixture of isomers) & $29350-73-0$ & Positive & Negative & INACTIVE & Known Negative & \\
\hline 23 & 1353 & 2-hexenal & $6728-26-3$ & Positive & Positive & PLAUSIBLE & Known Positive & \\
\hline 24 & 1364 & 2-pentenal & 764-39-6 & Positive & Positive & PLAUSIBLE & Known Positive & \\
\hline 25 & 1446 & 4-hydroxy-2,5-dimethyl-3(2H)-furanone & $3658-77-3$ & Positive & Positive & PROBABLE & Known Positive & \\
\hline 26 & 1449 & 2-ethyl-4-hydroxy-5-methyl-3(2H)-furanone & $27538-09-6$ & Positive & Positive & PLAUSIBLE & Negative & \\
\hline 27 & 1450 & 4-hydroxy-5-methyl-3(2H)-furanone & $19322-27-1$ & Positive & Positive & PROBABLE & Known Positive & Equivocal in Ono's data was revised (Table 1). \\
\hline 28 & 1456 & 2,5-dimethyl-4-oxo-3(5H)-furyl acetate & $4166-20-5$ & Positive & Positive & PLAUSIBLE & Negative & Ames test was newly conducted (Table 2). \\
\hline 29 & 1480 & maltol & $118-71-8$ & Positive & Positive & EQUIVOCAL & Known Positive & Ames test was newly conducted (Table 2). \\
\hline 30 & 1503 & 2-furyl methyl ketone & $1192-62-7$ & Positive & Negative & EQUIVOCAL & Known Positive & \\
\hline 31 & 1506 & 3-acetyl-2,5-dimethylfuran & 10599-70-9 & Positive & Positive & EQUIVOCAL & Known Positive & Ames test was newly conducted (Table 2). \\
\hline 32 & 1519 & 4,5-dihydro-2,5-dimethyl-4-oxofuran-3-yl butyrate & $114099-96-6$ & Positive & Positive & PLAUSIBLE & Negative & Ames test was newly conducted (Table 2). \\
\hline 33 & 1560 & allyl isothiocyanate & $57-06-7$ & Positive & Positive & PLAUSIBLE & Known Positive & $\begin{array}{l}\text { Equivocal in Ono's data was revised (Table 1). } \\
\text { Ames test was newly conducted (Table 2). }\end{array}$ \\
\hline 34 & 1561 & butyl isothiocyanate & $592-82-5$ & Positive & Positive & PLAUSIBLE & Known Positive & Equivocal in Ono's data was revised (Table 1). \\
\hline 35 & 1563 & phenethyl isothiocyanate & $2257-09-2$ & Positive & Positive & PLAUSIBLE & Known Positive & Equivocal in Ono's data was revised (Table 1). \\
\hline 36 & 1576 & ethyl 3-phenylglycidate & $121-39-1$ & Positive & Positive & PLAUSiBLE & Known Negative & \\
\hline 37 & 2100 & furfural propyleneglycol acetal & 4359-54-0 & Positive & Positive & INACTIVE & Negative & Ames test was newly conducted (Table 2). \\
\hline 38 & 2101 & furfuryl formate & $13493-97-5$ & Positive & Positive & EQUIVOCAL & Inconclusive & Ames test was newly conducted (Table 2). \\
\hline 39 & 2144 & methyl beta-phenylglycidate & $37161-74-3$ & Positive & Positive & PLAUSIBLE & Known Positive & Ames test was newly conducted (Table 2). \\
\hline 40 & 2157 & 6-methoxyquinoline & $5263-87-6$ & Positive & Positive & PROBABLE & Known Positive & Ames test was newly conducted (Table 2). \\
\hline 41 & - & 2-methylquinoline & $91-63-4$ & Positive & Positive & PLAUSIBLE & Known Positive & Ames test was newly conducted (Table 2). \\
\hline 42 & - & 4-methylquinoline & 491-35-0 & Positive & Positive & PROBABLE & Known Positive & Hansen database [6] \\
\hline 43 & - & quinoline & $91-22-5$ & Positive & Positive & PROBABLE & Known Positive & Hansen database [6] \\
\hline 44 & - & S-methyl methanethiosulfonate & 2949-92-0 & Positive & Negative & INACTIVE & Out of Domain & Ames test was newly conducted (Table 2). \\
\hline 45 & 3 & allyl hexanoate & $123-68-2$ & Negative & Negative & INACTIVE & Known Negative & \\
\hline 46 & 7 & allyl isovalerate & $2835-39-4$ & Negative & Negative & INACTIVE & Known Negative & \\
\hline 47 & 19 & allyl cinnamate & 1866-31-5 & Negative & Negative & INACTIVE & Inconclusive & \\
\hline 48 & 22 & benzaldehyde & $100-52-7$ & Negative & Negative & INACTIVE & Known Negative & \\
\hline 49 & 23 & benzyl acetate & $140-11-4$ & Negative & Negative & INACTIVE & Known Negative & \\
\hline 50 & 24 & benzyl benzoate & $120-51-4$ & Negative & Negative & INACTIVE & Negative & \\
\hline 51 & 25 & benzyl alcohol & $100-51-6$ & Negative & Negative & INACTIVE & Known Negative & \\
\hline 52 & 42 & isoamyl formate & $110-45-2$ & Negative & Negative & INACTIVE & Known Negative & \\
\hline 53 & 52 & isoamyl alcohol & $123-51-3$ & Negative & Negative & INACTIVE & Known Negative & \\
\hline 54 & 58 & geranyl acetate & $105-87-3$ & Negative & Negative & INACTIVE & Known Negative & \\
\hline 55 & 79 & formic acid & $64-18-6$ & Negative & Negative & INACTIVE & Known Negative & \\
\hline 56 & 80 & acetaldehyde & $75-07-0$ & Negative & Negative & INACTIVE & Known Negative & \\
\hline 57 & 81 & acetic acid & $64-19-7$ & Negative & Negative & INACTIVE & Known Negative & \\
\hline 58 & 82 & propyl alcohol & $71-23-8$ & Negative & Negative & INACTIVE & Known Negative & \\
\hline 59 & 83 & propionaldehyde & $123-38-6$ & Negative & Negative & INACTIVE & Known Negative & \\
\hline 60 & 84 & propionic acid & $79-09-4$ & Negative & Negative & INACTIVE & Known Negative & \\
\hline 61 & 85 & butyl alcohol & $71-36-3$ & Negative & Negative & INACTIVE & Known Negative & \\
\hline 62 & 86 & butyraldehyde & $123-72-8$ & Negative & Negative & INACTIVE & Known Negative & \\
\hline 63 & 87 & butyric acid & $107-92-6$ & Negative & Negative & INACTIVE & Known Negative & \\
\hline 64 & 88 & amyl alcohol & $71-41-0$ & Negative & Negative & INACTIVE & Known Negative & \\
\hline 65 & 92 & hexanal & $66-25-1$ & Negative & Negative & INACTIVE & Negative & \\
\hline 66 & 93 & hexanoic acid & $142-62-1$ & Negative & Negative & INACTIVE & Known Negative & \\
\hline 67 & 95 & heptanal & $111-71-7$ & Negative & Negative & INACTIVE & Known Negative & \\
\hline 68 & 96 & heptanoic acid & $111-14-8$ & Negative & Negative & INACTIVE & Known Negative & \\
\hline 69 & 97 & 1-octanol & $111-87-5$ & Negative & Negative & INACTIVE & Known Negative & \\
\hline
\end{tabular}


Table 4406 food flavor chemicals assessed by Ames test and QSARs (Continued)

\begin{tabular}{|c|c|c|c|c|c|c|c|c|}
\hline 70 & 98 & octanal & $124-13-0$ & Negative & Negative & INACTIVE & Negative & \\
\hline 71 & 99 & octanoic acid & $124-07-2$ & Negative & Negative & INACTIVE & Known Negative & \\
\hline 72 & 101 & nonanal & $124-19-6$ & Negative & Negative & INACTIVE & Known Negative & \\
\hline 73 & 104 & decanal & $112-31-2$ & Negative & Negative & INACTIVE & Known Negative & \\
\hline 74 & 105 & decanoic acid & $334-48-5$ & Negative & Negative & INACTIVE & Known Negative & \\
\hline 75 & 107 & undecanal & $112-44-7$ & Negative & Negative & INACTIVE & Negative & \\
\hline 76 & 109 & lauryl alcohol & $112-53-8$ & Negative & Negative & INACTIVE & Known Negative & \\
\hline 77 & 111 & lauric acid & $143-07-7$ & Negative & Negative & INACTIVE & Known Negative & \\
\hline 78 & 113 & myristic acid & $544-63-8$ & Negative & Negative & INACTIVE & Known Negative & \\
\hline 79 & 114 & 1-hexadecanol & $36653-82-4$ & Negative & Negative & INACTIVE & Known Negative & \\
\hline 80 & 116 & stearic acid & $57-11-4$ & Negative & Negative & INACTIVE & Known Negative & \\
\hline 81 & 125 & methyl acetate & $79-20-9$ & Negative & Negative & INACTIVE & Known Negative & \\
\hline 82 & 127 & butyl acetate & $123-86-4$ & Negative & Negative & INACTIVE & Known Negative & \\
\hline 83 & 128 & hexyl acetate & $142-92-7$ & Negative & Negative & INACTIVE & Negative & Ames test was newly conducted (Table 2). \\
\hline 84 & 139 & acetone & 67-64-1 & Negative & Negative & INACTIVE & Known Negative & \\
\hline 85 & 184 & butyl stearate & 123-95-5 & Negative & Negative & INACTIVE & Negative & \\
\hline 86 & 196 & ethyl isovalerate & $108-64-5$ & Negative & Negative & INACTIVE & Known Negative & \\
\hline 87 & 219 & 4-hydroxybutyric acid lactone & $96-48-0$ & Negative & Negative & INACTIVE & Known Negative & \\
\hline 88 & 225 & gamma-heptalactone & $105-21-5$ & Negative & Negative & INACTIVE & Negative & \\
\hline 89 & 229 & gamma-nonalactone & 104-61-0 & Negative & Negative & INACTIVE & Negative & \\
\hline 90 & 233 & gamma-undecalactone & $104-67-6$ & Negative & Negative & INACTIVE & Known Negative & \\
\hline 91 & 236 & delta-dodecalactone & 713-95-1 & Negative & Negative & INACTIVE & Negative & Ames test was newly conducted (Table 2). \\
\hline 92 & 239 & omega-pentadecalactone & $106-02-5$ & Negative & Negative & INACTIVE & Known Negative & \\
\hline 93 & 249 & $\begin{array}{l}\text { cis-4-hydroxy-6-dodecenoic acid lactone; 1,4-dodec-6- } \\
\text { enolactone }\end{array}$ & $18679-18-0$ & Negative & Negative & INACTIVE & Negative & \\
\hline 94 & 251 & isobutyl alcohol & 78-83-1 & Negative & Negative & INACTIVE & Known Negative & \\
\hline 95 & 252 & isobutyraldehyde & $78-84-2$ & Negative & Negative & INACTIVE & Known Negative & Equivocal in Ono's data was revised (Table 1). \\
\hline 96 & 253 & isobutyric acid & $79-31-2$ & Negative & Negative & INACTIVE & Negative & \\
\hline 97 & 254 & 2-methylbutyraldehyde & $96-17-3$ & Negative & Negative & INACTIVE & Known Negative & \\
\hline 98 & 255 & 2-methylbutyric acid & $116-53-0$ & Negative & Negative & INACTIVE & Known Negative & Ames test was newly conducted (Table 2). \\
\hline 99 & 256 & 2-ethylbutanal & 97-96-1 & Negative & Negative & INACTIVE & Negative & Ames test was newly conducted (Table 2). \\
\hline 100 & 258 & 3-methylbutyraldehyde & 590-86-3 & Negative & Negative & INACTIVE & Known Negative & \\
\hline 101 & 260 & 2-methylpentanal & 123-15-9 & Negative & Negative & INACTIVE & Negative & \\
\hline 102 & 267 & 2-ethyl-1-hexanol & $104-76-7$ & Negative & Negative & INACTIVE & Known Negative & \\
\hline 103 & 273 & 2,6-dimethyloctanal & 7779-07-9 & Negative & Negative & INACTIVE & Negative & \\
\hline 104 & 277 & isopropyl alcohol & $67-63-0$ & Negative & Negative & INACTIVE & Known Negative & \\
\hline 105 & 278 & 2-butanone & 78-93-3 & Negative & Negative & INACTIVE & Known Negative & \\
\hline 106 & 301 & 4-methyl-2-pentanone & 108-10-1 & Negative & Negative & INACTIVE & Known Negative & \\
\hline 107 & 302 & 2,6-dimethyl-4-heptanone & 108-83-8 & Negative & Negative & INACTIVE & Known Negative & \\
\hline 108 & 305 & isopropyl acetate & $108-21-4$ & Negative & Negative & INACTIVE & Known Negative & \\
\hline 109 & 311 & isopropyl myristate & $110-27-0$ & Negative & Negative & INACTIVE & Known Negative & \\
\hline 110 & 327 & (5or6)-decenoic acid & 72881-27-7 & Negative & Negative & INACTIVE & Negative & Ames test was newly conducted (Table 2). \\
\hline 111 & 333 & oleic Acid & $112-80-1$ & Negative & Negative & INACTIVE & Known Negative & \\
\hline 112 & 346 & methyl linoleate & $112-63-0$ & Negative & Negative & INACTIVE & Known Positive & \\
\hline 113 & 349 & 2,6-dimethyl-5-heptenal & $106-72-9$ & Negative & Negative & INACTIVE & Negative & \\
\hline 114 & 356 & linalool & $78-70-6$ & Negative & Negative & INACTIVE & Known Negative & \\
\hline 115 & 359 & linalyl acetate & $115-95-7$ & Negative & Negative & INACTIVE & Known Positive & \\
\hline 116 & 366 & alpha-terpineol & $98-55-5$ & Negative & Negative & INACTIVE & Known Negative & \\
\hline 117 & 374 & p-menth-8-en-1-ol; $\beta$-terpineol & $138-87-4$ & Negative & Negative & INACTIVE & Negative & \\
\hline 118 & 380 & carvone & $99-49-0$ & Negative & Negative & INACTIVE & Known Negative & \\
\hline 119 & 381 & carveol & $99-48-9$ & Negative & Negative & INACTIVE & Known Negative & \\
\hline 120 & 382 & carvyl acetate & $97-42-7$ & Negative & Negative & INACTIVE & Known Negative & \\
\hline 121 & 388 & alpha-ionone & $127-41-3$ & Negative & Negative & INACTIVE & Known Negative & \\
\hline 122 & 389 & beta-ionone & $79-77-6$ & Negative & Negative & INACTIVE & Known Negative & \\
\hline 123 & 398 & methyl-alpha-ionone & $127-42-4$ & Negative & Negative & INACTIVE & Known Negative & \\
\hline 124 & 400 & methyl-delta-ionone & 7784-98-7 & Negative & Negative & INACTIVE & Known Negative & \\
\hline 125 & 405 & acetoin & $513-86-0$ & Negative & Negative & INACTIVE & Known Negative & \\
\hline 126 & 413 & 3,4-hexanedione & 4437-51-8 & Negative & Positive & PLAUSIBLE & Known Positive & \\
\hline 127 & 418 & methylcyclo- pentenolone & $80-71-7$ & Negative & Negative & INACTIVE & Known Negative & \\
\hline 128 & 424 & 2-hydroxy-2-cyclohexen-1-one & $10316-66-2$ & Negative & Negative & PROBABLE & Negative & \\
\hline 129 & 427 & menthol & $89-78-1$ & Negative & Negative & INACTIVE & Known Negative & \\
\hline 130 & 443 & I-menthol ethylene glycol carbonate & $156324-78-6$ & Negative & Negative & INACTIVE & Negative & \\
\hline 131 & 444 & (-)-menthol 1- and 2-propylene glycol carbonate & $156329-82-2$ & Negative & Negative & INACTIVE & Negative & \\
\hline 132 & 446 & dl-menthone 1,2-glycerol ketal & 63187-91-7 & Negative & Negative & INACTIVE & Negative & \\
\hline 133 & 452 & dimethyl sulfide & $75-18-3$ & Negative & Negative & INACTIVE & Out of Domain & Ames test was newly conducted (Table 2). \\
\hline 134 & 458 & allyl sulfide & 592-88-1 & Negative & Negative & INACTIVE & Negative & \\
\hline 135 & 492 & methylthio 2-(acetyloxy)propionate & 74586-09-7 & Negative & Negative & INACTIVE & Negative & \\
\hline 136 & 493 & methylthio 2-(propionyloxy) propionate & $827024-53-3$ & Negative & Negative & INACTIVE & Negative & \\
\hline 137 & 520 & 2-mercaptopinane & $23832-18-0$ & Negative & Negative & INACTIVE & Out of Domain & Ames test was newly conducted (Table 2). \\
\hline 138 & 521 & allyl mercaptan & 870-23-5 & Negative & Negative & INACTIVE & Out of Domain & \\
\hline 139 & 525 & benzenethiol & 108-98-5 & Negative & Negative & INACTIVE & Known Positive & \\
\hline 140 & 526 & benzyl mercaptan & $100-53-8$ & Negative & Negative & INACTIVE & Known Negative & \\
\hline 141 & 532 & 1,2-ethanedithiol & $540-63-6$ & Negative & Negative & INACTIVE & Known Negative & \\
\hline
\end{tabular}


Table 4406 food flavor chemicals assessed by Ames test and QSARs (Continued)

\begin{tabular}{|c|c|c|c|c|c|c|c|c|}
\hline 142 & 551 & 2-mercaptopropionic acid & $79-42-5$ & Negative & Negative & INACTIVE & Negative & \\
\hline 143 & 564 & dimethyl disulfide & $624-92-0$ & Negative & Negative & INACTIVE & Known Negative & \\
\hline 144 & 572 & allyl disulfide & $2179-57-9$ & Negative & Negative & INACTIVE & Negative & \\
\hline 145 & 578 & phenyl disulfide & $882-33-7$ & Negative & Negative & INACTIVE & Known Negative & \\
\hline 146 & 579 & benzyl disulfide & $150-60-7$ & Negative & Negative & INACTIVE & Known Positive & \\
\hline 147 & 595 & ethyl acetoacetate & $141-97-9$ & Negative & Positive & INACTIVE & Known Negative & \\
\hline 148 & 610 & hydroxycitronellol & $107-74-4$ & Negative & Negative & INACTIVE & Negative & \\
\hline 149 & 611 & hydroxycitronellal & $107-75-5$ & Negative & Negative & INACTIVE & Negative & \\
\hline 150 & 612 & hydroxycitronellal dimethyl acetal & $141-92-4$ & Negative & Negative & INACTIVE & Negative & \\
\hline 151 & 614 & diethyl malonate & $105-53-3$ & Negative & Negative & INACTIVE & Known Negative & \\
\hline 152 & 616 & dimethyl succinate & $106-65-0$ & Negative & Negative & INACTIVE & Known Negative & \\
\hline 153 & 618 & fumaric acid & $110-17-8$ & Negative & Negative & INACTIVE & Known Negative & \\
\hline 154 & 619 & I-malic acid & $97-67-6$ & Negative & Negative & INACTIVE & Known Negative & \\
\hline 155 & 623 & adipic acid & $124-04-9$ & Negative & Negative & INACTIVE & Known Negative & \\
\hline 156 & 625 & dibutyl sebacate & $109-43-3$ & Negative & Negative & INACTIVE & Known Negative & \\
\hline 157 & 626 & ethylene brassylate & $105-95-3$ & Negative & Negative & INACTIVE & Known Negative & \\
\hline 158 & 627 & aconitic acid & $499-12-7$ & Negative & Negative & INACTIVE & Known Negative & \\
\hline 159 & 645 & 3-phenylpropionaldehyde & $104-53-0$ & Negative & Negative & INACTIVE & Negative & \\
\hline 160 & 647 & cinnamyl alcohol & $104-54-1$ & Negative & Negative & INACTIVE & Known Negative & \\
\hline 161 & 657 & cinnamic acid & $621-82-9$ & Negative & Negative & INACTIVE & Known Negative & \\
\hline 162 & 659 & ethyl cinnamate & $103-36-6$ & Negative & Negative & INACTIVE & Known Negative & \\
\hline 163 & 667 & cyclohexyl cinnamate & 7779-17-1 & Negative & Negative & INACTIVE & Negative & \\
\hline 164 & 670 & benzyl cinnamate & $103-41-3$ & Negative & Negative & INACTIVE & Negative & \\
\hline 165 & 674 & alpha-amylcinnamyl alcohol & $101-85-9$ & Negative & Negative & INACTIVE & Negative & \\
\hline 166 & 683 & alpha-methylcinnamaldehyde & $101-39-3$ & Negative & Negative & INACTIVE & Known Negative & \\
\hline 167 & 685 & alpha-amylcinnamaldehyde & $122-40-7$ & Negative & Negative & INACTIVE & Known Negative & \\
\hline 168 & 686 & alpha-hexylcinnamaldehyde & $101-86-0$ & Negative & Negative & INACTIVE & Positive & \\
\hline 169 & 688 & o-methoxycinnamaldehyde & $1504-74-1$ & Negative & Negative & PLAUSIBLE & Known Positive & \\
\hline 170 & 689 & p-methoxy-alpha-methyl-cinnamaldehyde & $65405-67-6$ & Negative & Negative & INACTIVE & Positive & \\
\hline 171 & 690 & phenol & $108-95-2$ & Negative & Negative & INACTIVE & Known Negative & Equivocal in Ono's data was revised (Table 1). \\
\hline 172 & 691 & o-cresol & $95-48-7$ & Negative & Negative & INACTIVE & Known Negative & \\
\hline 173 & 692 & m-cresol & $108-39-4$ & Negative & Negative & INACTIVE & Known Negative & \\
\hline 174 & 693 & p-cresol & $106-44-5$ & Negative & Negative & INACTIVE & Known Negative & \\
\hline 175 & 694 & p-ethylphenol & $123-07-9$ & Negative & Negative & INACTIVE & Known Negative & \\
\hline 176 & 706 & 2,5-xylenol & $95-87-4$ & Negative & Negative & INACTIVE & Known Negative & \\
\hline 177 & 707 & 2,6-xylenol & $576-26-1$ & Negative & Negative & INACTIVE & Known Negative & \\
\hline 178 & 708 & 3,4-xylenol & $95-65-8$ & Negative & Negative & INACTIVE & Known Negative & \\
\hline 179 & 709 & thymol & $89-83-8$ & Negative & Negative & INACTIVE & Known Negative & \\
\hline 180 & 713 & guaiacol & $90-05-1$ & Negative & Negative & INACTIVE & Known Negative & \\
\hline 181 & 721 & 2,6-dimethoxyphenol & $91-10-1$ & Negative & Negative & INACTIVE & Known Negative & \\
\hline 182 & 725 & 4-ethenyl-2-methoxyphenol & $7786-61-0$ & Negative & Negative & INACTIVE & Known Negative & Ames test was newly conducted (Table 2). \\
\hline 183 & 727 & 2-hydroxyacetophenone & $118-93-4$ & Negative & Negative & INACTIVE & Negative & \\
\hline 184 & 733 & 4-(1,1-dimethylethyl) phenol & 98-54-4 & Negative & Negative & INACTIVE & Known Negative & \\
\hline 185 & 736 & phenyl salicylate & $118-55-8$ & Negative & Positive & INACTIVE & Known Negative & \\
\hline 186 & 738 & furfuryl alcohol & $98-00-0$ & Negative & Negative & INACTIVE & Known Negative & Equivocal in Ono's data was revised (Table 1). \\
\hline 187 & 744 & furfural & $98-01-1$ & Negative & Negative & EQUIVOCAL & Known Negative & Equivocal in Ono's data was revised (Table 1). \\
\hline 188 & 745 & 5-methylfurfural & $620-02-0$ & Negative & Negative & EQUIVOCAL & Known Negative & Ames test was newly conducted (Table 2). \\
\hline 189 & 753 & pulegone & $89-82-7$ & Negative & Negative & INACTIVE & Known Positive & \\
\hline 190 & 758 & menthofuran & 494-90-6 & Negative & Negative & EQUIVOCAL & Known Negative & \\
\hline 191 & 761 & 2-methylpyrazine & $109-08-0$ & Negative & Negative & INACTIVE & Known Negative & \\
\hline 192 & 762 & 2-ethylpyrazine & $13925-00-3$ & Negative & Negative & INACTIVE & Known Negative & \\
\hline 193 & 765 & 2,3-dimethylpyrazine & $5910-89-4$ & Negative & Negative & INACTIVE & Known Negative & \\
\hline 194 & 766 & 2,5-dimethylpyrazine & $123-32-0$ & Negative & Negative & INACTIVE & Known Negative & \\
\hline 195 & 768 & 2-ethyl-3-methylpyrazine & $15707-23-0$ & Negative & Negative & INACTIVE & Negative & \\
\hline 196 & 774 & 2,3,5-trimethylpyrazine & $14667-55-1$ & Negative & Negative & INACTIVE & Known Negative & \\
\hline 197 & 775 & 2-ethyl-3 (5 or 6)-dimethylpyrazine & $13925-07-0$ & Negative & Negative & INACTIVE & Negative & \\
\hline 198 & 780 & 2,3,5,6-tetramethylpyrazine & $1124-11-4$ & Negative & Negative & INACTIVE & Negative & \\
\hline 199 & 788 & 2-methoxy-3-methylpyrazine & $2847-30-5$ & Negative & Negative & INACTIVE & Negative & \\
\hline 200 & 798 & 5-methylquinoxaline & $13708-12-8$ & Negative & Negative & INACTIVE & Negative & \\
\hline 201 & 799 & alpha-methylbenzyl alcohol & $98-85-1$ & Negative & Negative & INACTIVE & Known Negative & \\
\hline 202 & 806 & acetophenone & $98-86-2$ & Negative & Negative & INACTIVE & Known Negative & \\
\hline 203 & 811 & methyl beta-naphthyl ketone & $93-08-3$ & Negative & Negative & INACTIVE & Known Negative & \\
\hline 204 & 812 & 4-acetyl-6-t-butyl-1,1-dimethylindan & $13171-00-1$ & Negative & Negative & INACTIVE & Known Negative & \\
\hline 205 & 818 & 4-(p-methoxyphenyl)-2-butanone & $104-20-1$ & Negative & Negative & INACTIVE & Negative & \\
\hline 206 & 819 & 4-phenyl-3-buten-2-ol & $17488-65-2$ & Negative & Negative & INACTIVE & Negative & \\
\hline 207 & 824 & propiophenone & $93-55-0$ & Negative & Negative & INACTIVE & Negative & \\
\hline 208 & 825 & alpha-propylphenethyl alcohol & 705-73-7 & Negative & Negative & INACTIVE & Negative & \\
\hline 209 & 826 & 1-(p-methoxyphenyl)-1-penten-3-one & $104-27-8$ & Negative & Negative & INACTIVE & Negative & \\
\hline 210 & 831 & benzophenone & $119-61-9$ & Negative & Negative & INACTIVE & Known Negative & \\
\hline 211 & 833 & 1-Phenyl-1,2-propanedione & $579-07-7$ & Negative & Negative & INACTIVE & Known Negative & \\
\hline 212 & 834 & ethyl benzoylacetate & $94-02-0$ & Negative & Negative & INACTIVE & Negative & \\
\hline 213 & 841 & benzyl formate & $104-57-4$ & Negative & Negative & INACTIVE & Inconclusive & \\
\hline 214 & 850 & benzoic acid & $65-85-0$ & Negative & Negative & INACTIVE & Known Negative & \\
\hline
\end{tabular}


Table 4406 food flavor chemicals assessed by Ames test and QSARs (Continued)

\begin{tabular}{|c|c|c|c|c|c|c|c|c|}
\hline 215 & 851 & methyl benzoate & $93-58-3$ & Negative & Negative & INACTIVE & Known Negative & \\
\hline 216 & 857 & isoamyl benzoate & $94-46-2$ & Negative & Negative & INACTIVE & Negative & \\
\hline 217 & 864 & p-isopropylbenzyl alcohol & $536-60-7$ & Negative & Negative & INACTIVE & Negative & \\
\hline 218 & 866 & 4-methylbenzaldehyde & $104-87-0$ & Negative & Negative & INACTIVE & Known Negative & Ames test was newly conducted (Table 2). \\
\hline 219 & 867 & tolualdehydes (mixed ortho, meta, para) & $529-20-4$ & Negative & Negative & INACTIVE & Known Negative & \\
\hline 220 & 868 & cuminaldehyde & $122-03-2$ & Negative & Negative & INACTIVE & Negative & \\
\hline 221 & 870 & butyl 4-hydroxybenzoate & $94-26-8$ & Negative & Negative & INACTIVE & Known Negative & \\
\hline 222 & 871 & anisyl alcohol & $105-13-5$ & Negative & Negative & INACTIVE & Known Negative & \\
\hline 223 & 877 & veratraldehyde & $120-14-9$ & Negative & Negative & INACTIVE & Known Negative & \\
\hline 224 & 878 & p-methoxybenzaldehyde & $123-11-5$ & Negative & Negative & INACTIVE & Known Negative & \\
\hline 225 & 879 & p-ethoxybenzaldehyde & $10031-82-0$ & Negative & Negative & INACTIVE & Negative & \\
\hline 226 & 884 & methyl anisate & $121-98-2$ & Negative & Negative & INACTIVE & Negative & \\
\hline 227 & 888 & vanillyl butyl ether & $82654-98-6$ & Negative & Negative & INACTIVE & Known Negative & \\
\hline 228 & 889 & vanillin & $121-33-5$ & Negative & Negative & INACTIVE & Known Negative & \\
\hline 229 & 893 & ethyl vanillin & $121-32-4$ & Negative & Negative & INACTIVE & Known Negative & \\
\hline 230 & 894 & piperonyl acetate & $326-61-4$ & Negative & Negative & INACTIVE & Known Negative & \\
\hline 231 & 896 & piperonal & $120-57-0$ & Negative & Negative & INACTIVE & Known Positive & \\
\hline 232 & 897 & salicyladehyde & $90-02-8$ & Negative & Negative & INACTIVE & Known Negative & \\
\hline 233 & 899 & methyl salicylate & $119-36-8$ & Negative & Negative & INACTIVE & Known Negative & \\
\hline 234 & 909 & glycerol & $56-81-5$ & Negative & Negative & INACTIVE & Known Negative & \\
\hline 235 & 918 & glyceryl monostearate & $123-94-4$ & Negative & Negative & INACTIVE & Known Negative & \\
\hline 236 & 925 & propylene glycol & $57-55-6$ & Negative & Negative & INACTIVE & Known Negative & \\
\hline 237 & 928 & hexanal propyleneglycol acetal & $1599-49-1$ & Negative & Negative & INACTIVE & Negative & Ames test was newly conducted (Table 2). \\
\hline 238 & 930 & lactic acid & $598-82-3$ & Negative & Negative & INACTIVE & Known Negative & \\
\hline 239 & 931 & ethyl lactate & $97-64-3$ & Negative & Negative & INACTIVE & Known Negative & \\
\hline 240 & 935 & butyl butyryllactate & 7492-70-8 & Negative & Negative & INACTIVE & Negative & \\
\hline 241 & 936 & pyruvic acid & $127-17-3$ & Negative & Negative & INACTIVE & Known Negative & \\
\hline 242 & 938 & ethyl pyruvate & $617-35-6$ & Negative & Positive & INACTIVE & Known Negative & \\
\hline 243 & 941 & acetaldehyde diethyl acetal & $105-57-7$ & Negative & Negative & INACTIVE & Known Positive & Ames test was newly conducted (Table 2). \\
\hline 244 & 951 & pyrazine & $290-37-9$ & Negative & Negative & INACTIVE & Known Negative & \\
\hline 245 & 953 & ethyl vanillin isobutyrate & 188417-26-7 & Negative & Negative & INACTIVE & Negative & \\
\hline 246 & 973 & p-mentha-1,8-dien-7-al & $2111-75-3$ & Negative & Negative & INACTIVE & Known Negative & \\
\hline 247 & 977 & 2,6,6-trimethylcyclohexa-1,3-dienyl methanal & $116-26-7$ & Negative & Negative & INACTIVE & Inconclusive & \\
\hline 248 & 987 & phenethyl alcohol & $60-12-8$ & Negative & Negative & INACTIVE & Known Negative & \\
\hline 249 & 1002 & phenylacetaldehyde & $122-78-1$ & Negative & Negative & INACTIVE & Negative & \\
\hline 250 & 1007 & phenylacetic acid & $103-82-2$ & Negative & Negative & INACTIVE & Known Negative & \\
\hline 251 & 1009 & ethyl phenylacetate & $101-97-3$ & Negative & Negative & INACTIVE & Negative & \\
\hline 252 & 1013 & isobutyl phenylacetate & $102-13-6$ & Negative & Negative & INACTIVE & Known Negative & \\
\hline 253 & 1014 & isoamyl phenylacetate & $102-19-2$ & Negative & Negative & INACTIVE & Negative & \\
\hline 254 & 1023 & p-tolylacetaldehyde & $104-09-6$ & Negative & Negative & INACTIVE & Negative & \\
\hline 255 & 1027 & ethyl (p-tolyloxy)acetate & $67028-40-4$ & Negative & Negative & INACTIVE & Negative & \\
\hline 256 & 1028 & 2-phenoxyethyl isobutyrate & $103-60-6$ & Negative & Negative & INACTIVE & Negative & \\
\hline 257 & 1029 & sodium 2-(4-methoxyphenoxy)propanoate & $13794-15-5$ & Negative & Negative & INACTIVE & Negative & \\
\hline 258 & 1031 & 2-(4-methyl-5-thiazolyl)ethanol & $137-00-8$ & Negative & Negative & INACTIVE & Negative & Ames test was newly conducted (Table 2). \\
\hline 259 & 1035 & 4,5-dimethylthiazole & 3581-91-7 & Negative & Negative & INACTIVE & Out of Domain & \\
\hline 260 & 1043 & 4-methylthiazole & 693-95-8 & Negative & Negative & INACTIVE & Known Negative & \\
\hline 261 & 1050 & 5-methyl-2-thiophenecarboxaldehyde & 13679-70-4 & Negative & Negative & INACTIVE & Negative & \\
\hline 262 & 1072 & 2-furanmethanethiol & $98-02-2$ & Negative & Negative & INACTIVE & Negative & Ames test was newly conducted (Table 2). \\
\hline 263 & 1094 & cyclohexyl butyrate & $1551-44-6$ & Negative & Negative & INACTIVE & Known Negative & \\
\hline 264 & 1100 & cyclohexanone & $108-94-1$ & Negative & Negative & INACTIVE & Known Negative & \\
\hline 265 & 1101 & cyclopentanone & $120-92-3$ & Negative & Negative & INACTIVE & Negative & \\
\hline 266 & 1106 & 2-hexylidene cyclopentanone & $17373-89-6$ & Negative & Negative & INACTIVE & Negative & \\
\hline 267 & 1108 & 2,2,6-trimethylcyclohexanone & $2408-37-9$ & Negative & Negative & INACTIVE & Negative & \\
\hline 268 & 1111 & tetramethyl ethylcyclohexenone (mixture of isomers) & $17369-60-7$ & Negative & Negative & INACTIVE & Negative & \\
\hline 269 & 1112 & isophorone & 78-59-1 & Negative & Negative & INACTIVE & Known Negative & \\
\hline 270 & 1120 & 6-methyl-5-hepten-2-one & $110-93-0$ & Negative & Negative & INACTIVE & Negative & \\
\hline 271 & 1124 & 3-penten-2-one & $625-33-2$ & Negative & Positive & INACTIVE & Negative & \\
\hline 272 & 1131 & 4-methyl-3-penten-2-one & $141-79-7$ & Negative & Negative & INACTIVE & Known Negative & \\
\hline 273 & 1134 & 6-methyl-3,5-heptadien-2-one & $1604-28-0$ & Negative & Negative & INACTIVE & Inconclusive & \\
\hline 274 & 1135 & (E)-7-methyl-3-octen-2-one & $33046-81-0$ & Negative & Negative & INACTIVE & Negative & \\
\hline 275 & 1153 & 1-decen-3-ol & $51100-54-0$ & Negative & Negative & INACTIVE & Negative & \\
\hline 276 & 1164 & $\begin{array}{l}(+/-)-(2,6,6, \text {-trimethyl-2-hydroxycyclohexylidene)acetic } \\
\text { acid gamma-lactone }\end{array}$ & $15356-74-8$ & Negative & Negative & INACTIVE & Negative & \\
\hline 277 & 1166 & octahydrocoumarin & 4430-31-3 & Negative & Negative & INACTIVE & Negative & \\
\hline 278 & 1171 & dihydrocoumarin & $119-84-6$ & Negative & Negative & INACTIVE & Known Negative & \\
\hline 279 & 1172 & 6-methylcoumarin & $92-48-8$ & Negative & Negative & INACTIVE & Known Negative & Equivocal in Ono's data was revised (Table 1) \\
\hline 280 & 1185 & 2,4-nonadienal & 6750-03-4 & Negative & Negative & INACTIVE & Positive & \\
\hline 281 & 1186 & nona-2-trans-6-cis-dienal & $557-48-2$ & Negative & Negative & INACTIVE & Known Negative & \\
\hline 282 & 1190 & 2-trans,4-trans-decadienal & $25152-84-5$ & Negative & Negative & INACTIVE & Known Negative & \\
\hline 283 & 1193 & ethyl 2,4,7-decatrienoate & $78417-28-4$ & Negative & Negative & INACTIVE & Negative & \\
\hline 284 & 1199 & (+/--)-2-methyl-1-butanol & $137-32-6$ & Negative & Negative & INACTIVE & Negative & \\
\hline 285 & 1209 & 2-methyl-2-pentenal & $623-36-9$ & Negative & Negative & PLAUSible & Positive & \\
\hline 286 & 1219 & dl-citronellol & $106-22-9$ & Negative & Negative & INACTIVE & Known Negative & \\
\hline
\end{tabular}


Table 4406 food flavor chemicals assessed by Ames test and QSARs (Continued)

\begin{tabular}{|c|c|c|c|c|c|c|c|c|}
\hline 287 & 1220 & citronellal & $106-23-0$ & Negative & Negative & INACTIVE & Known Negative & \\
\hline 288 & 1223 & geraniol & $106-24-1$ & Negative & Negative & INACTIVE & Known Negative & \\
\hline 289 & 1225 & citral & $5392-40-5$ & Negative & Negative & INACTIVE & Known Negative & \\
\hline 290 & 1230 & farnesol & $4602-84-0$ & Negative & Negative & INACTIVE & Known Negative & \\
\hline 291 & 1234 & eucalyptol & $470-82-6$ & Negative & Negative & INACTIVE & Known Negative & \\
\hline 292 & 1241 & anisole & $100-66-3$ & Negative & Negative & INACTIVE & Negative & \\
\hline 293 & 1243 & p-methylanisole & 104-93-8 & Negative & Negative & INACTIVE & Negative & \\
\hline 294 & 1244 & p-propylanisole & $104-45-0$ & Negative & Negative & INACTIVE & Negative & \\
\hline 295 & 1248 & 1,2-dimethoxybenzene & $91-16-7$ & Negative & Negative & INACTIVE & Negative & \\
\hline 296 & 1249 & m-dimethoxybenzene & $151-10-0$ & Negative & Negative & INACTIVE & Negative & \\
\hline 297 & 1250 & p-dimethoxybenzene & $150-78-7$ & Negative & Negative & INACTIVE & Known Negative & \\
\hline 298 & 1255 & diphenyl ether & $101-84-8$ & Negative & Negative & INACTIVE & Known Negative & \\
\hline 299 & 1256 & dibenzyl ether & $103-50-4$ & Negative & Negative & INACTIVE & Known Negative & \\
\hline 300 & 1257 & beta-naphthyl methyl ether & $93-04-9$ & Negative & Negative & INACTIVE & Inconclusive & \\
\hline 301 & 1258 & beta-naphthyl ethyl ether & $93-18-5$ & Negative & Negative & INACTIVE & Known Negative & \\
\hline 302 & 1259 & beta-naphthyl isobutyl ether & $2173-57-1$ & Negative & Negative & INACTIVE & Negative & \\
\hline 303 & 1260 & isoeugenol & $97-54-1$ & Negative & Negative & INACTIVE & Known Negative & \\
\hline 304 & 1263 & isoeugenyl phenylacetate & $120-24-1$ & Negative & Negative & INACTIVE & Negative & \\
\hline 305 & 1264 & propenylguaethol & $94-86-0$ & Negative & Negative & INACTIVE & Negative & \\
\hline 306 & 1266 & isoeugenyl methyl ether & $93-16-3$ & Negative & Negative & INACTIVE & Negative & Ames test was newly conducted (Table 2). \\
\hline 307 & 1268 & isoeugenyl benzyl ether & $120-11-6$ & Negative & Negative & INACTIVE & Negative & \\
\hline 308 & 1289 & 3-mercapto-2-methylbutan-1-ol & 227456-33-9 & Negative & Negative & INACTIVE & Negative & \\
\hline 309 & 1301 & indole & $120-72-9$ & Negative & Negative & INACTIVE & Known Positive & Ames test was newly conducted (Table 2). \\
\hline 310 & 1303 & isoquinoline & $119-65-3$ & Negative & Positive & INACTIVE & Known Negative & \\
\hline 311 & 1304 & skatole & $83-34-1$ & Negative & Negative & INACTIVE & Known Negative & Ames test was newly conducted (Table 2). \\
\hline 312 & 1314 & pyrrole & $109-97-7$ & Negative & Negative & INACTIVE & Known Negative & \\
\hline 313 & 1315 & 3-ethylpyridine & $536-78-7$ & Negative & Negative & INACTIVE & Negative & \\
\hline 314 & 1316 & 3-acetylpyridine & $350-03-8$ & Negative & Negative & INACTIVE & Negative & \\
\hline 315 & 1323 & camphene & $79-92-5$ & Negative & Negative & INACTIVE & Known Negative & \\
\hline 316 & 1324 & beta-caryophyllene & $87-44-5$ & Negative & Negative & INACTIVE & Known Negative & \\
\hline 317 & 1325 & p-cymene & $99-87-6$ & Negative & Negative & INACTIVE & Negative & \\
\hline 318 & 1326 & d-limonene & $5989-27-5$ & Negative & Negative & INACTIVE & Known Negative & \\
\hline 319 & 1327 & myrcene & $123-35-3$ & Negative & Negative & INACTIVE & Negative & \\
\hline 320 & 1329 & alpha-pinene & $80-56-8$ & Negative & Negative & INACTIVE & Known Negative & \\
\hline 321 & 1330 & beta-pinene & $127-91-3$ & Negative & Negative & INACTIVE & Known Negative & \\
\hline 322 & 1332 & biphenyl & $92-52-4$ & Negative & Negative & INACTIVE & Known Negative & \\
\hline 323 & 1334 & 4-methylbiphenyl & $644-08-6$ & Negative & Negative & INACTIVE & Negative & \\
\hline 324 & 1335 & ' & $90-12-0$ & Negative & Negative & INACTIVE & Known Negative & \\
\hline 325 & 1340 & p-mentha-1,4-diene & $99-85-4$ & Negative & Negative & INACTIVE & Negative & Ames test was newly conducted (Table 2). \\
\hline 326 & 1341 & $1,3,5$-undecatriene & $16356-11-9$ & Negative & Negative & INACTIVE & Negative & Ames test was newly conducted (Table 2). \\
\hline 327 & 1351 & ethyl acrylate & $140-88-5$ & Negative & Negative & INACTIVE & Known Negative & \\
\hline 328 & 1354 & 2-hexenol & 2305-21-7 & Negative & Negative & INACTIVE & Negative & Ames test was newly conducted (Table 2). \\
\hline 329 & 1356 & methyl 2-nonynoate & $111-80-8$ & Negative & Negative & INACTIVE & Out of Domain & \\
\hline 330 & 1357 & methyl 2-octynoate & $111-12-6$ & Negative & Negative & INACTIVE & Out of Domain & \\
\hline 331 & 1360 & 2-heptenal & $18829-55-5$ & Negative & Negative & PLAUSIBLE & Known Negative & \\
\hline 332 & 1362 & 2-nonenal & 2463-53-8 & Negative & Negative & INACTIVE & Inconclusive & \\
\hline 333 & 1363 & 2-octenal & $2363-89-5$ & Negative & Negative & INACTIVE & Inconclusive & \\
\hline 334 & 1371 & (E)-2-butenoic acid & $107-93-7$ & Negative & Negative & INACTIVE & Negative & \\
\hline 335 & 1385 & borneol & $507-70-0$ & Negative & Negative & INACTIVE & Known Negative & \\
\hline 336 & 1391 & isobornyl propionate & $2756-56-1$ & Negative & Negative & INACTIVE & Negative & \\
\hline 337 & 1395 & d-camphor & 464-49-3 & Negative & Negative & INACTIVE & Known Negative & \\
\hline 338 & 1408 & 3-L-menthoxypropane-1,2-diol & 87061-04-9 & Negative & Negative & INACTIVE & Known Negative & \\
\hline 339 & 1411 & 3-(I-menthoxy)-2-methylpropane-1,2-diol & $195863-84-4$ & Negative & Negative & INACTIVE & Negative & \\
\hline 340 & 1413 & d,I-menthol 1-and 2-propylene glycol carbonate & $30304-82-6$ & Negative & Negative & INACTIVE & Negative & \\
\hline 341 & 1416 & p-menthane-3,8-diol & $42822-86-6$ & Negative & Negative & INACTIVE & Negative & \\
\hline 342 & 1441 & 2-(3-phenylpropyl)tetrahydrofuran & $3208-40-0$ & Negative & Negative & INACTIVE & Negative & \\
\hline 343 & 1443 & tetrahydrofurfuryl alcohol & $97-99-4$ & Negative & Negative & INACTIVE & Known Negative & \\
\hline 344 & 1445 & tetrahydrofurfuryl propionate & $637-65-0$ & Negative & Positive & INACTIVE & Negative & \\
\hline 345 & 1451 & 4-methoxy-2,5-dimethyl-3(2H)-furanone & 4077-47-8 & Negative & Negative & PLAUSIBLE & Negative & Ames test was newly conducted (Table 2). \\
\hline 346 & 1454 & linalool oxide (furanoid) & $1365-19-1$ & Negative & Negative & INACTIVE & Negative & Ames test was newly conducted (Table 2). \\
\hline 347 & 1459 & beta-methylphenethyl alcohol & $1123-85-9$ & Negative & Negative & INACTIVE & Negative & \\
\hline 348 & 1467 & 2-phenylpropionaldehyde & $93-53-8$ & Negative & Negative & INACTIVE & Negative & \\
\hline 349 & 1468 & 2-phenylpropionaldehyde dimethyl acetal & $90-87-9$ & Negative & Negative & INACTIVE & Negative & \\
\hline 350 & 1470 & 2-phenylpropyl isobutyrate & 65813-53-8 & Negative & Negative & INACTIVE & Negative & \\
\hline 351 & 1472 & 5-methyl-2-phenyl-2-hexenal & $21834-92-4$ & Negative & Negative & INACTIVE & Inconclusive & Ames test was newly conducted (Table 2). \\
\hline 352 & 1487 & 2-methylfuran & $534-22-5$ & Negative & Negative & EQUIVOCAL & Known Negative & \\
\hline 353 & 1488 & 2,5-dimethylfuran & $625-86-5$ & Negative & Negative & EQUIVOCAL & Known Negative & \\
\hline 354 & 1494 & 3-methyl-2-(3-methylbut-2-enyl)-furan & $15186-51-3$ & Negative & Negative & INACTIVE & Negative & \\
\hline 355 & 1497 & 3-(2-furyl)acrolein & $623-30-3$ & Negative & Negative & PLAUSIBLE & Known Negative & \\
\hline 356 & 1511 & 4-(2-furyl)-3-buten-2-one & $623-15-4$ & Negative & Negative & INACTIVE & Known Negative & \\
\hline 357 & 1513 & ethyl 3-(2-furyl)propanoate & $10031-90-0$ & Negative & Positive & INACTIVE & Negative & \\
\hline 358 & 1526 & O-ethyl S-(2-furylmethyl)thiocarbonate & $376595-42-5$ & Negative & Positive & INACTIVE & Negative & \\
\hline 359 & 1529 & eugenol & $97-53-0$ & Negative & Negative & INACTIVE & Known Negative & \\
\hline
\end{tabular}


Table 4406 food flavor chemicals assessed by Ames test and QSARs (Continued)

\begin{tabular}{|c|c|c|c|c|c|c|c|c|}
\hline 360 & 1534 & methyl anthranilate & $134-20-3$ & Negative & Negative & INACTIVE & Known Negative & \\
\hline 361 & 1535 & ethyl anthranilate & $87-25-2$ & Negative & Negative & INACTIVE & Known Negative & \\
\hline 362 & 1536 & butyl anthranilate & 7756-96-9 & Negative & Negative & INACTIVE & Known Negative & \\
\hline 363 & 1537 & isobutyl anthranilate & $7779-77-3$ & Negative & Negative & INACTIVE & Known Negative & \\
\hline 364 & 1540 & linalyl anthranilate & $7149-26-0$ & Negative & Negative & INACTIVE & Known Negative & \\
\hline 365 & 1541 & cyclohexyl anthranilate & $7779-16-0$ & Negative & Negative & INACTIVE & Known Negative & \\
\hline 366 & 1543 & phenylethyl anthranilate & $133-18-6$ & Negative & Negative & INACTIVE & Known Negative & \\
\hline 367 & 1545 & methyl N-methylanthranilate & $85-91-6$ & Negative & Negative & INACTIVE & Known Negative & \\
\hline 368 & 1549 & methyl $\mathrm{N}$-formylanthranilate & $41270-80-8$ & Negative & Negative & INACTIVE & Negative & \\
\hline 369 & 1552 & $\mathrm{~N}$-benzoylanthranilic acid & 579-93-1 & Negative & Negative & INACTIVE & Negative & \\
\hline 370 & 1562 & benzyl isothiocyanate & $622-78-6$ & Negative & Negative & PLAUSIBLE & Known Positive & \\
\hline 371 & 1575 & beta-caryophyllene oxide & $1139-30-6$ & Negative & Negative & INACTIVE & Negative & \\
\hline 372 & 1577 & ethyl methylphenylglycidate & $77-83-8$ & Negative & Negative & INACTIVE & Known Negative & \\
\hline 373 & 1579 & ethylamine & $75-04-7$ & Negative & Negative & INACTIVE & Known Negative & \\
\hline 374 & 1581 & isopropylamine & $75-31-0$ & Negative & Negative & INACTIVE & Known Negative & \\
\hline 375 & 1582 & butylamine & $109-73-9$ & Negative & Negative & INACTIVE & Known Negative & \\
\hline 376 & 1583 & isobutylamine & 78-81-9 & Negative & Negative & INACTIVE & Known Negative & \\
\hline 377 & 1584 & sec-butylamine & $13952-84-6$ & Negative & Negative & INACTIVE & Known Negative & \\
\hline 378 & 1585 & pentylamine & $110-58-7$ & Negative & Negative & INACTIVE & Known Negative & \\
\hline 379 & 1592 & acetamide & $60-35-5$ & Negative & Positive & INACTIVE & Known Negative & \\
\hline 380 & 1595 & 2-isopropyl-N,2,3-trimethylbutyramide & $51115-67-4$ & Negative & Negative & INACTIVE & Negative & \\
\hline 381 & 1598 & $\mathrm{~N}$-isobutyl (E,E)-2,4-decadienamide & $18836-52-7$ & Negative & Negative & INACTIVE & Negative & \\
\hline 382 & 1600 & piperine & $94-62-2$ & Negative & Negative & INACTIVE & Known Negative & \\
\hline 383 & 1607 & piperidine & $110-89-4$ & Negative & Negative & INACTIVE & Negative & \\
\hline 384 & 1609 & pyrrolidine & $123-75-1$ & Negative & Negative & INACTIVE & Known Positive & \\
\hline 385 & 1610 & trimethylamine & $75-50-3$ & Negative & Negative & INACTIVE & Known Negative & \\
\hline 386 & 1611 & triethylamine & $121-44-8$ & Negative & Negative & INACTIVE & Known Negative & \\
\hline 387 & 1615 & piperazine & $110-85-0$ & Negative & Negative & INACTIVE & Known Negative & \\
\hline 388 & 1649 & 1-phenyl-3-methyl-3-pentanol & $10415-87-9$ & Negative & Negative & INACTIVE & Negative & \\
\hline 389 & 1654 & alpha,alpha-dimethylphenethyl formate & $10058-43-2$ & Negative & Negative & INACTIVE & Inconclusive & \\
\hline 390 & 1681 & allyl thiohexanoate & $156420-69-8$ & Negative & Negative & INACTIVE & Negative & \\
\hline 391 & 1687 & 3,6-diethyl-1,2,4,5-tetrathiane & $54717-12-3$ & Negative & Negative & INACTIVE & Negative & \\
\hline 392 & 1700 & allyl propyl disulfide & 2179-59-1 & Negative & Negative & INACTIVE & Known Negative & \\
\hline 393 & 1716 & dihydroxyacetone dimer & $62147-49-3$ & Negative & Positive & INACTIVE & Known Positive & \\
\hline 394 & 1767 & $\mathrm{~N}$-(heptan-4-y|)benzo[d][1,3]dioxole-5-carboxamide & $745047-51-2$ & Negative & Negative & INACTIVE & Negative & \\
\hline 395 & 1768 & N1-(2,4-dimethoxybenzy))-N2-(2-(pyridin-2- & $745047-53-4$ & Negative & Negative & INACTIVE & Negative & \\
\hline 396 & 1772 & Whegthydolitylalamidelamine & 686298-93-1 & Negative & Positive & INACTIVE & Negative & \\
\hline 397 & 1774 & N-lactoyl ethanolamine & $5422-34-4$ & Negative & Negative & INACTIVE & Known Positive & \\
\hline 398 & 1776 & $\mathrm{~N}$-[(ethoxycarbonyl)methyl)-p-menthane-3- & $68489-14-5$ & Negative & Negative & INACTIVE & Negative & Equivocal in Ono's data was revised (Table 1). \\
\hline 399 & 1777 & 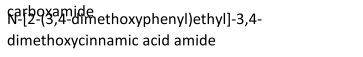 & $69444-90-2$ & Negative & Negative & INACTIVE & Negative & \\
\hline 400 & 1853 & 2-(1-menthoxy)ethanol & 38618-23-4 & Negative & Negative & INACTIVE & Negative & Ames test was newly conducted (Table 2). \\
\hline 401 & 1882 & vanillin propyleneglycol acetal & $68527-74-2$ & Negative & Negative & INACTIVE & Negative & Ames test was newly conducted (Table 2). \\
\hline 402 & 1894 & 5-hexenyl isothiocyanate & $49776-81-0$ & Negative & Negative & PLAUSIBLE & Inconclusive & Ames test was newly conducted (Table 2). \\
\hline 403 & 2141 & butyl 2-naphthyl ether & $10484-56-7$ & Negative & Negative & INACTIVE & Negative & Ames test was newly conducted (Table 2). \\
\hline 404 & - & 2-butoxyethyl acetate & $112-07-2$ & Negative & Negative & INACTIVE & Negative & Ames test was newly conducted (Table 2). \\
\hline 405 & - & 2,4-dimethyl-4-phenyltetrahydrofuran & $82461-14-1$ & Negative & Positive & INACTIVE & Negative & Ames test was newly conducted (Table 2). \\
\hline 406 & . & 2-methyl-2-butanethiol & $1679-09-0$ & Negative & Negative & INACTIVE & Out of Domain & Ames test was newly conducted (Table 2). \\
\hline
\end{tabular}

the training set. Because the accuracy of the prototype depends on the training data set and the data splitting process is not replicable, 80 prototypes were built to search for the best model. The prototypes that earned favorable prediction scores were selected for further performance evaluation by using the Ames test data of flavoring chemicals, and their performances were compared with those of the benchmarks. Finally, a new
QSAR model "StarDrop NIHS 834_67" was developed. The prediction result is ranked as "positive" or "negative."

\section{Performance of the QSAR model}

We evaluated the performance of StarDrop NIHS834_67 to predict the Ames mutagenicity. We calculated the Ames mutagenicity of 406 food flavors listed in the new

Table 5 Results of QSAR calculation of 406 flavor chemicals in 2X2 contingency matrix

\begin{tabular}{|c|c|c|c|c|c|c|c|c|}
\hline & & \multicolumn{2}{|c|}{ StarDrop NIHS 834_67 } & \multicolumn{2}{|c|}{ Derek Nexus 6.1.0 } & \multicolumn{3}{|c|}{ CASE Ultra 1.8.0.2 GT1_BMUT } \\
\hline & & $\bar{P}$ & $\mathbf{N}$ & $P$ & $\mathbf{N}$ & $P$ & $\mathbf{N}$ & OOD \\
\hline \multirow[t]{2}{*}{ Ames test result } & $P$ & 35 & 9 & 31 & 13 & 31 & 12 & 1 \\
\hline & $\mathrm{N}$ & 13 & 349 & 14 & 348 & 28 & 327 & 7 \\
\hline
\end{tabular}


Table 6 Performance of three QSARs for predicting Ames mutagenicity of 406 flavor chemicals

\begin{tabular}{lllll}
\hline & Sensitivity (\%) & Specificity (\%) & Accuracy (\%) & Applicability (\%) \\
\hline StarDrop NIHS 834_67 & 79.5 & 96.4 & 94.6 & 100.0 \\
Derek Nexus 6.1.0 & 70.5 & 96.1 & 93.3 & 100.0 \\
CASE Ultra 1.8.0.2 GT1_BMUT & 70.5 & 90.3 & 88.2 & 98.0 \\
\hline
\end{tabular}

Table 7 Ames positive chemicals, but predicted as negative by StarDrop NIHS 834 67 (False negative)

\begin{tabular}{|c|c|c|c|c|c|c|}
\hline No. & JECFA No. & Chemical Name & CAS No. & Structure & Substructure Class & Note \\
\hline 1 & 429 & menthone & $89-80-5$ & & Ketones & $\begin{array}{l}\text { DEREK: INACTIVE } \\
\text { CASE Ultra: Known Negative }\end{array}$ \\
\hline 2 & 656 & trans-cinnamaldehyde & $104-55-2$ & & Aromatic aldehydes & $\begin{array}{l}\text { DEREK: PLAUSIBLE } \\
\text { CASE Ultra: Known Positive }\end{array}$ \\
\hline 3 & 728 & raspberry ketone & $5471-51-2$ & & Ketones & $\begin{array}{l}\text { DEREK: INACTIVE } \\
\text { CASE Ultra: Negative }\end{array}$ \\
\hline 4 & 767 & 2,6-dimethylpyrazine & $108-50-9$ & & Newly designated flavors & $\begin{array}{l}\text { DEREK: INACTIVE } \\
\text { CASE Ultra: Known Positive }\end{array}$ \\
\hline 5 & 820 & 4-phenyl-3-buten-2-one & $122-57-6$ & & Ketones & $\begin{array}{l}\text { DEREK: INACTIVE } \\
\text { CASE Ultra: Known Positive }\end{array}$ \\
\hline 6 & 1208 & 4-methyl-2-pentenal & $5362-56-1$ & & Aliphatic higher aldehydes & $\begin{array}{l}\text { DEREK: PLAUSIBLE } \\
\text { CASE Ultra: Positive }\end{array}$ \\
\hline 7 & 1346 & cadinene (mixture of isomers) & $29,350-73-0$ & & Terpene hydrocarbons & $\begin{array}{l}\text { DEREK: INACTIVE } \\
\text { CASE Ultra: Known Negative }\end{array}$ \\
\hline 8 & 1503 & 2-Furyl methyl ketone & $1192-62-7$ & & Ketones & $\begin{array}{l}\text { DEREK: EQUIVOCAL } \\
\text { CASE Ultra: Known Positive }\end{array}$ \\
\hline 9 & - & S-methyl methanethiosulfonate & 2949-92-0 & & Esters & $\begin{array}{l}\text { DEREK: INACTIVE } \\
\text { CASE Ultra: Out of Domain }\end{array}$ \\
\hline
\end{tabular}


Table 8 Ames negative chemicals, but predicted as positive by StarDrop NIHS 834_67 (False positive)

\begin{tabular}{|c|c|c|c|c|c|c|}
\hline No. & $\begin{array}{l}\text { JECFA } \\
\text { No. }\end{array}$ & Chemical Name & CAS No. & Structure & $\begin{array}{l}\text { Substructure } \\
\text { Class }\end{array}$ & Note \\
\hline 1 & 413 & 3,4-hexanedione & $\begin{array}{l}4437-51- \\
8\end{array}$ & & Ketones & $\begin{array}{l}\text { DEREK: PLAUSIBLE } \\
\text { CASE Ultra: Known } \\
\text { Positive }\end{array}$ \\
\hline 2 & 595 & ethyl acetoacetate & 141-97-9 & & Esters & $\begin{array}{l}\text { DEREK: INACTIVE } \\
\text { CASE Ultra: Known } \\
\text { Negative }\end{array}$ \\
\hline 3 & 736 & phenyl salicylate & $118-55-8$ & & Esters & $\begin{array}{l}\text { DEREK: INACTIVE } \\
\text { CASE Ultra: Known } \\
\text { Negative }\end{array}$ \\
\hline 4 & 938 & ethyl pyruvate & $617-35-6$ & & Esters & $\begin{array}{l}\text { DEREK: INACTIVE } \\
\text { CASE Ultra: Known } \\
\text { Negative }\end{array}$ \\
\hline 5 & 1124 & 3-penten-2-one & $625-33-2$ & & Ketones & $\begin{array}{l}\text { DEREK: INACTIVE } \\
\text { CASE Ultra: Negative }\end{array}$ \\
\hline 6 & 1303 & isoquinoline & $119-65-3$ & & $\begin{array}{l}\text { Newly designated } \\
\text { flavors }\end{array}$ & $\begin{array}{l}\text { DEREK: INACTIVE } \\
\text { CASE Ultra: Known } \\
\text { Negative }\end{array}$ \\
\hline 7 & 1445 & $\begin{array}{l}\text { tetrahydrofurfuryl } \\
\text { propionate }\end{array}$ & $637-65-0$ & & Esters & $\begin{array}{l}\text { DEREK: INACTIVE } \\
\text { CASE Ultra: Negative }\end{array}$ \\
\hline 8 & 1513 & ethyl 3-(2-furyl)propanoate & $\begin{array}{l}10,031- \\
90-0\end{array}$ & & Esters & $\begin{array}{l}\text { DEREK: INACTIVE } \\
\text { CASE Ultra: Negative }\end{array}$ \\
\hline 9 & 1526 & $\begin{array}{l}\text { O-ethyl S-(2- } \\
\text { furylmethyl)thiocarbonate }\end{array}$ & $\begin{array}{l}376,595- \\
42-5\end{array}$ & & Esters & $\begin{array}{l}\text { DEREK: INACTIVE } \\
\text { CASE Ultra: Negative }\end{array}$ \\
\hline 10 & 1592 & acetamide & $60-35-5$ & & Not classified & $\begin{array}{l}\text { DEREK: INACTIVE } \\
\text { CASE Ultra: Known } \\
\text { Negative }\end{array}$ \\
\hline 11 & 1716 & dihydroxyacetone dimer & $\begin{array}{l}62,147- \\
49-3\end{array}$ & & Ketones & $\begin{array}{l}\text { DEREK: INACTIVE } \\
\text { CASE Ultra: Known } \\
\text { Positive }\end{array}$ \\
\hline 12 & 1772 & N-gluconyl ethanolamine & $\begin{array}{l}686,298- \\
93-1\end{array}$ & & Not classified & $\begin{array}{l}\text { DEREK: INACTIVE } \\
\text { CASE Ultra: Negative }\end{array}$ \\
\hline
\end{tabular}


Table 8 Ames negative chemicals, but predicted as positive by StarDrop NIHS 834_67 (False positive) (Continued)

\begin{tabular}{llllll}
\hline $\begin{array}{l}\text { No. JECFA } \\
\text { No. }\end{array}$ & Chemical Name & CAS No. & Structure & $\begin{array}{l}\text { Substructure } \\
\text { Class }\end{array}$ & Note \\
\hline $13-$ & 2-butoxyethyl acetate & $112-07-2$ & Esters & $\begin{array}{l}\text { DEREK: INACTIVE } \\
\text { CASE Ultra: Negative }\end{array}$ \\
\hline
\end{tabular}

Ames test database by using StarDrop NIHS 834_67, DEREK Nexus $^{\text {TM }}$, and CASE Ultra. Table 4 shows the results of the QSAR calculation. Table 5 is a $2 \times 2$ prediction matrix, and Table 6 shows the performance (sensitivity, specificity, accuracy, and applicability) of the three (Q) SARs. StarDrop NIHS 834_67 showed the best performance. Table 7 shows nine FN chemicals that were positive in the Ames test but were negatively predicted by NIHS834_67. Table 8 shows 13 FP chemicals that were negative in the Ames test but were positively predicted by NIHS834_67.

\section{Discussion}

We have developed new Ames database consisting of 406 types of food flavor chemicals. This benchmark food flavor chemicals database is open to the public and useful for risk assessment of food additives and developing QSAR models for predicting Ames mutagenicity of food flavor chemicals and other low molecular weight chemicals. The main body of the database is derived from the database reported by Ono et al. [4]. We re-assessed 14 "equivocal" chemicals and classified them as negative, positive, or inconclusive. However, the positive and negative chemicals remaining in Ono's database were not re-assessed. Some of these chemicals may also be misjudged. In fact, 2,3-pentanedione (600-14-6), which was negative in Ono's database, was clearly positive in the present Ames test (Additional file (6)). To ensure database robustness, it is necessary to re-assess the test results reported as positive and negative. As will be described later, especially, the results of the Ames test that differ from the QSAR prediction results could be questioned.

In 2012, Ono et al. reported the performance of three commercial QSAR tools (Derek for Windows, MultiCASE, and ADMEWorks) for predicting Ames mutagenicity of 367 food flavor chemicals [4]. Derek for Windows and MultiCASE are earlier models of DEREK Nexus $^{\mathrm{TM}}$ and CASE Ultra, respectively. As a result, the sensitivity, specificity, and accuracy were $38.9,93.4$, and 88.0\% (Derek for Windows), 25.0, 94.3, and 87.5\% (MultiCASE), respectively. In this study, we evaluated the performance of DEREK Nexus ${ }^{\mathrm{TM}}$ and CASE Ultra for 406 food flavors in the new Ames database. As a result, the sensitivity, specificity, and accuracy were 70.5, 96.1, and 93.3\% (DEREK Nexus ${ }^{\mathrm{TM}}$ ) and 70.5, 90.3, and 88.2\% (CASE Ultra), respectively. These results indicate that the performance of the QSAR prediction has improved significantly over the last decade. The improvement in sensitivity was particularly remarkable. Improvement of the QSAR models and accumulation of newly acquired Ames test training data may have contributed to the high performance. In particular, the NIHS-sponsored Ames/QSAR International Challenge Project has contributed significantly to improving the performance of commercial QSAR tools, such as DEREK Nexus ${ }^{\mathrm{TM}}$ and CASE Ultra, which have acquired over 12,000 unique chemical Ames datasets [24]. The newly developed StarDrop NIHS 834_67 outperformed DEREK Nexus ${ }^{\text {TM }}$ and CASE Ultra. StarDrop NIHS 834_67 also acquired 428 chemicals (positive: 255, negative: 173) selected from the 12,000 unique chemical Ames datasets. Despite incorporating the same training data, StarDrop NIHS 834_67 provided higher prediction, probably due to differences in the target chemical space. Flavor chemicals are relatively low molecular weight and have unique functional groups that allow them to focus on the chemical space of interest and develop highly predictable models with relatively small size training data. Our attempt to develop a local QSAR model that focused on flavor chemicals has been somewhat successful. However, it is not surprising that that StarDrop NIHS 834_67 showed higher performance than other QSAR tools. It may be because StarDrop NIHS 834_67 used the results of 39 new flavor chemical datasets and revised existing flavor chemical data for training and validation data.

Considering that the estimated interlaboratory reproducibility of the Ames test has been reported to be approximately $85 \%[27,28]$, the performance of the prediction may be approaching the upper limit. Nonetheless, FN and FP analysis points to improvements in the database and QSAR models. Of the nine FN flavor chemicals by StarDrop NIHS 834_67, menthone (8980-5), raspberry ketone (54-51-2), and cadinene (29350-73-0) were also predicted as negative by DEREK Nexus $^{\mathrm{TM}}$ and CASE Ultra (Table 7). The Ames mutagenicity of these chemicals, which were predicted to be negative by the three QSARs, may actually be negative chemicals. We need to perform actual Ames tests to confirm.

In this study, we examined the Ames tests for raspberry ketone (54-51-2) and the result was positive (Table 4). However, the mutagenic activity was very weak (RAV: 10) (Additional file (12)). Structural features 
found in FN chemicals include the $\alpha, \beta$-unsaturated carbonyl structures, trans-cinnamaldehyde (104-55-2), 4phenyl-3-buten-2-one (122-57-6), 4-methyl-2-pentenal (5362-56-1), and 2- furyl methyl ketone (1192-62-7), which were predicted to be positive by DEREK Nexus ${ }^{\mathrm{Tm}}$ and/or CASE Ultra. The $\alpha, \beta$-unsaturated carbonyl structure is a typical alert for Ames mutagenicity [2931]. These predictions indicate that the alert is incorporated in DEREK Nexus ${ }^{\text {Th }}$ and CASE Ultra but not in StarDrop NIHS 834_67. By incorporating $\alpha$ and $\beta$ unsaturated carbonyl chemicals as training data, it is expected that the FN rate of StarDrop NIHS 834_67 will be reduced and the predictability will be improved.

On the other hand, of the 13 FP chemicals, 3,4-hexanedione (4437-51-8) was also predicted as positive by DEREK Nexus ${ }^{\text {Tw }}$ and CASE Ultra. The Ames mutagenicity of this chemical may actually be positive. Interestingly, 12 other FP flavor chemicals were correctly predicted as negative by DEREK Nexus ${ }^{\mathrm{Tm}}$ and CASE Ultra, which highlights the different characteristics between StarDrop NIHS 834_67 and other QSAR tools and indicates the potential for further improvement.

\section{Conclusions}

We developed a new Ames database of 406 food flavor chemicals. Using this database and other Ames datasets of chemicals that are structurally similar to flavor chemicals, we also developed a new QSAR model for predicting Ames mutagenicity. The local QSAR model, StarDrop NIHS 834_67, is customized to efficiently predict the mutagenicity of food flavors and other low molecular weight chemicals, delivering performance superior to that of other commercial QSAR tools. By further improving the model, it can be used to assess the mutagenicity of food flavors without actual testing.

\section{Abbreviations}

QSAR: Quantitative structure-activity relationship; TP: True positive; TN: True negative; FP: False positive; FN: False negative

\section{Supplementary Information}

The online version contains supplementary material available at https://doi. org/10.1186/s41021-021-00182-6.

\section{Additional file 1: Raw data for the Ames tests.}

\section{Acknowledgments}

We appreciate the companies and the Japan Flavor and Fragrant Materials Association for providing flavor chemicals for the Ames test.

\section{Authors' contributions}

Conceived and designed the studies: MH, QSAR model development: ST and MK, QSAR calculation: AK, Analyzed the data: TK, Ames test management: KS, MY, MY, KM, and KH, Wrote the paper: MH and TK. The authors read and approved the final manuscript.

\section{Funding}

This work was supported by the Ministry of Health, Labor, and Welfare under Grant Numbers H30-Food-General-003 and H30-Chemistry-Destination-005 and by the Japan Agency for Medical Research and Development under Grant Number 20am0101123j0104.

Availability of data and materials

All generated data are included in this manuscript. Raw data for the Ames tests are available in the Additional files.

\section{Declarations}

Ethics approval and consent to participate

Not applicable.

\section{Consent for publication}

Not applicable.

\section{Competing interests}

The authors declare that they have no competing interests.

\section{Author details}

${ }^{1}$ Division of Genetics and Mutagenesis, National Institute of Health Sciences, Kawasaki city, Kanagawa, Japan. ${ }^{2}$ HULINKS Inc., Chuo city, Tokyo, Japan. ${ }^{3}$ Department of Applied Chemistry, National Defense Academy, Yokosuka city, Kanagawa, Japan. ${ }^{4}$ Division of General Affairs, National Institute of Health Sciences, Kawasaki City, Kanagawa, Japan.

Received: 28 December 2020 Accepted: 24 February 2021

Published online: 30 April 2021

\section{References}

1. Honma M. Threshold of toxicological concern for genotoxic impurities in pharmaceuticals. In: Nohmi T, Fukushima S, editors. Thresholds of genotoxic carcinogens. UK: Academic Press; 2016. p. 103-15.

2. Mortelmans K, Zeiger E. The Ames Salmonella/microsome mutagenicity assay. Mutat Res. 2000;455:29-60.

3. Honma M. An assessment of mutagenicity of chemical substances by (quantitative) structure-activity relationship. Genes Environ. 2020;42:23.

4. Ono A, Takahashi M, Hirose A, Kamata E, Kawamura T, Yamazaki T, Sato K, Yamada M, Fukumoto T, Okamura H, Mirokuji Y, Honma M. Validation of the (Q) SAR combination approach for mutagenicity prediction of flavor chemicals. Food Chem Toxicol. 2012;50:1538-46.

5. Okamura $\mathrm{H}$, Abe $\mathrm{H}$, Hasegawa-Baba $\mathrm{Y}$, et al. The Japan flavour and fragrance materials Association's (JFFMA) safety assessment of acetal food flavouring substances uniquely used in Japan. Food Addit Contam Part A Chem Anal Control Expo Risk Assess. 2015:32:1384-96.

6. Hansen K, Mika S, Schroeter T, et al. Benchmark data set for in Silico prediction of Ames mutagenicity. J Chem Inf Model. 2009;49:2077-81.

7. Mutagenicity test in under the industrial safety and health act. Test guideline and GLP (in Japanese). Tokyo: Japan Industrial Safety \& Health Association (JISHA); 1991.

8. OECD. Guideline for Testing of Chemicals Test Guideline No. 471: bacterial reverse mutation test. Paris: OECD; 1997. https://www.oecd.org/chemicalsa fety/risk-assessment/1948418.pdf

9. Williams RV, Amberg A, Brigo A, Coquin L, Giddings A, Glowienke S, Greene N, Jolly R, Kemper R, O'Leary-Steele C, Parenty A, Spirkl HP, Stalford SA, Weiner SK, Wichard J. It's difficult, but important, to make negative predictions. Regul Toxicol Pharmacol. 2016;76:79-86.

10. Barber C, Cayley A, Hanser T, Harding A, Heghes C, Vessey JD, Werner S, Weiner SK, Wichard J, Giddings A, Glowienke S, Parenty A, Brigo A, Spirkl HP, Amberg A, Kemper R, Greene N. Evaluation of a statistics-based Ames mutagenicity QSAR model and interpretation of the results obtained. Regul Toxicol Pharmacol. 2016;76:7-20.

11. Klopman G, Frierson MR, Rosenkranz HS. The structural basis of the mutagenicity of chemicals in Salmonella typhimurium: the gene-Tox data base. Mutat Res 1990;228(1):1-50.

12. Landry C, Kim MT, Kruhlak NL, Cross KP, Saiakhov R, Chakravarti S, Stavitskaya L. Transitioning to composite bacterial mutagenicity models in ICH M7 (Q) SAR analyses. Regul Toxicol Pharmacol. 2019;109:104488. 
13. McMahon RE, Cline JC, Thompson CZ. Assay of 855 test chemicals in ten tester strains using a new modification of the Ames test for bacterial mutagens. Cancer Res. 1979;39:682-93.

14. Gocke E, King MT, Eckhardt K, Wild D. Mutagenicity of cosmetics ingredients licensed by the European Communities. Mutat Res. 1981;90:91-109.

15. WHO Food Additives Series 59. Safety evaluation of certain food additives. 2008.

16. Zeiger $\mathrm{E}$, Haworth $\mathrm{S}$, Tests with a preincubation modification of the Salmonella/microsome assay. Progress in Mutation Research (J. Ashbby, F.J. Serres et al. Eds.), Vol. 5, World Health Organization. 1985.

17. WHO Food Additives Series 52. Safety evaluation of certain food additives and contaminants. 2004.

18. Wild D, King MT, Gocke E, Eckhardt K. Study of artificial flavouring substances for mutagenicity in the Salmonella/microsome, Basc and micronucleus tests. Food Chem Toxicol. 1983:21:707-19.

19. Kurttio P, Kalliokoski P, Lampelo S, Jantunen MJ. Mutagenic compounds in wood-chip drying fumes. Mutat Res. 1990;242:9-15.

20. Hiramoto K, Sekiguchi K, Ayuha K, et al. DNA breaking activity and mutagenicity of soy sauce: characterization of the active components and identification of 4-hydroxy-5-methyl-3(2H)-furanone. Mutat Res. 1996;359: $119-32$.

21. WHO Food Additives Series 56 . Safety evaluation of certain food additives. 2006.

22. Kassie F, Knasmuller S. Genotoxic effects of allyl isothiocyanate (AITC) and phenethyl isothiocyanate (PEITC). Chem Biol Interact. 2000;127:163-80.

23. Yamaguchi T. Mutagenicity of Isothiocyanates, Isocyanates and Thioureas on Salmonella typhimurium. Agric Biol Chem. 1980;44:3017-8.

24. Honma M, Kitazawa A, Cayley A, Williams RV, Barber C, Hanser T, Saiakhov R, Chakravarti S, Myatt GJ, Cross KP, Benfenati E, Raitano G, Mekenyan O, Petkov P, Bossa C, Benigni R, Battistelli CL, Giuliani A, Tcheremenskaia O, DeMeo C, Norinder U, Koga H, Jose C, Jeliazkova N, Kochev N, Paskaleva V Yang C, Daga PR, Clark RD, Rathman J. Improvement of quantitative structure-activity relationship (QSAR) tools for predicting Ames mutagenicity: outcomes of the Ames/QSAR International Challenge Project. Mutagenesis. 2019;34:3-16.

25. Honma M, Kitazawa A, Kasamatsu T, Sugiyama Kl. Screening for Ames mutagenicity of food flavor chemicals by (quantitative) structure-activity relationship. Genes Environ. 2020;42:32.

26. Butina D. Unsupervised Data Base clustering based on Daylight's fingerprint and Tanimoto similarity: a fast and automated way to cluster small and large data set. J Chem Inf Comput Sci. 1999;39:747-50.

27. Piegorsch WW, Zeiger E. Measuring intra-assay agreement for the Ames Salmonella assay. In: Hothorn L, editor. Lecture notes inMedical informatics, vol. 43. Heidelberg: Springer; 1991. p. 35-41.

28. Kamber M, Fluckiger-Isler S, Engelhardt G, Jaeckh R, Zeiger E. Comparison of the Ames II and traditional Ames test responses with respect to mutagenicity, strain specificities, need for metabolism and correlation with rodent carcinogenicity. Mutagenesis. 2009;24:359-66

29. Eder E, Hoffman C, Bastian H, Deininger C, Scheckenbach S. Molecular mechanisms of DNA damage initiated by alpha, beta-unsaturated carbonyl compounds as criteria for genotoxicity and mutagenicity. Environ Health Perspect. 1990;88:99-106.

30. Koleva YK, Madden JC, Cronin MT. Formation of categories from structureactivity relationships to allow read-across for risk assessment: toxicity of alpha,beta-unsaturated carbonyl compounds. Chem Res Toxicol. 2008;21: 2300-12.

31. Benigni R, Bossa C. Mechanisms of chemical carcinogenicity and mutagenicity: a review with implications for predictive toxicology. Chem Rev. 2011;111:2507-36.

\section{Publisher's Note}

Springer Nature remains neutral with regard to jurisdictional claims in published maps and institutional affiliations.

Ready to submit your research? Choose BMC and benefit from:

- fast, convenient online submission

- thorough peer review by experienced researchers in your field

- rapid publication on acceptance

- support for research data, including large and complex data types

- gold Open Access which fosters wider collaboration and increased citations

- maximum visibility for your research: over $100 \mathrm{M}$ website views per year

At $\mathrm{BMC}$, research is always in progress.

Learn more biomedcentral.com/submissions 